\title{
1 Initiation of ensemble kinesin-3 motility is regulated by the rigidity of \\ 2 cargo-motor attachment
}

4 Prakash Lama ${ }^{1,2}$ and Minhajuddin Sirajuddin ${ }^{1, \#}$

5

6 1. Centre for Cardiovascular Biology and Disease, Institute for Stem Cell Science and

7 Regenerative Medicine, GKVK Campus, Bengaluru - 560065, India

8 2. Manipal Academy of Higher Education, Manipal, India

9 \# Address correspondence to M. Sirajuddin: minhaj@instem.res.in

\section{Abstract}

12 Intracellular cargo transport is powered by molecular motors that move on their

13 respective filamentous tracks. A key component in this process is the tether between

14 cargo and motor, which is often connected by long slender coiled-coils. Several studies

15 have identified mechanisms that regulate cargo transport and can be broadly categorized

16 into regulation of the motor ATPase activity by autoinhibition, cargo adapters and

17 modifications in the cytoskeletal tracks. The regulatory effects of cargo-motor linkers

18 have been described in kinesin-3 subfamily motors. However, the effects of cargo-motor

19 linker rigidity on ensemble cargo transport has not been explored. Here we have built a

20 DNA origami scaffold, which can be tethered with multiple kinesin-3 motors using either

21 single or double-stranded DNA linkages, mimicking rigid versus flexible cargo-motor

22 linkages. Using this system, we show that regardless of the motor numbers attached to the

23 cargo, only linkers with a lesser degree of freedom allow motors to engage with

24 microtubule tracks. Together, our work identifies that the rigidity of cargo-motor linkages

25 influences motor motility. This opens up the possibilities to identify new factors that can

26 influence the rigidity of cargo-motor linkages that in turn can regulate intracellular cargo

27 transport. 


\section{Introduction}

32 Molecular motors belonging to the kinesin superfamily require three important components for their cellular function; the motor domain, coiled-coil helices and the terminal cargo binding domain (Sweeney and Holzbaur, 2018). The kinesin motor domain interacts with the microtubules, which in turn stimulates ATP turnover and the ATP hydrolyzing activity is converted into the mechanical force required for motility. Modulation of ATPase activity (Gennerich and Vale, 2009), sequestration of the motor domain (Hammond et al., 2010; Imanishi et al., 2006; Toropova et al., 2017; Ren et al., 2018) and modifications in the microtubule tracks (Sirajuddin et al., 2014; Lessard et al., 2019; McKenney et al., 2016; Monroy et al., 2020) are well-known regulatory mechanisms that govern kinesin motility. The cargo binding domain of a typical kinesin motor is located at the distal end of the motor domain. In many cases, the sequestration of motor domain is mediated by the cargo binding domain leading to an autoinhibitory state.

44 This autoinhibition can be relieved by cognate cargo adapters, thereby ensuring fidelity 45 and minimizing futile ATPase cycles during intracellular transport (Siddiqui and Straube, 2017). The third component in kinesin molecular motors are the tethers that connect motor domain and cargo binding domain. These tethers are often dimeric coiled-coil helices that span several hundreds of amino acids, thus rendering a single kinesin molecular motor dimeric in nature (Hirokawa et al., 2009b). The slender coiled coil domain also contains several breaks making them flexible, thereby providing several degrees of freedom to the kinesin motor during cargo transport.

Among the kinesin superfamily of motors, members of the kinesin-3 subfamily such as

54 KIF1A, KIF13B and KIF16 are known to be regulated by the flexible parts of the coiled 55 coil domain (Soppina et al., 2014). Using single molecule and engineered kinesin-3

56 motors it was inferred that the flexibility of the hinge region between NC and CC1

57 domains is important for dimerization and subsequent motility of kinesin-3 motors

58 (Soppina et al., 2014; Huo et al., 2012). The dimerization requirement for kinesin-3

59 processive motility was a contentious notion (Okada and Hirokawa, 1999; Hirokawa et

60 al., 2009a), which has been sufficiently addressed by several independent studies

61 (Soppina et al., 2014; Hammond et al., 2009; Tomishige et al., 2002). The current model 
62 suggests that dimerization of kinesin-3 motors leads to super-processive motility i.e.,

63 their ability to walk long distances along the microtubules (Soppina et al., 2014;

64 Scarabelli et al., 2015; Siddiqui and Straube, 2017). The super-processive property of

65 kinesin-3 motors have been attributed to the lysine rich loop (K-loop) in the motor

66 domain (Soppina and Verhey, 2014). The basic charge of K-loop mediates electrostatic

67 interaction with the acidic carboxy-terminal tails of tubulin, thus increasing the

68 processive motility of kinesin-3 motors. A recent study has also shown that

69 multimerization of kinesin-3 monomers can also result in cargo transport (Schimert et al.,

70 2019). While tremendous progress has been achieved in understanding kinesin-3 motors

71 and their motility at the single-molecule level, the regulatory aspects of multiple kinesin-

723 motors have not been explored. In fact, the conventional method to study molecular

73 motors and their regulation has been an endeavor that majorly involves purified

74 molecular motors and studying them at the single-molecule level. However, molecular

75 motors often work in teams with varying motor ensemble numbers. To study motor

76 ensemble properties and probe the importance of the cargo-motor linker rigidity in

77 ensemble conditions we designed a synthetic scaffold system. The DNA origami scaffold

78 developed in this study can accommodate up to 28 motors, far exceeding the ensemble

79 numbers achieved in previous studies (Toropova et al., 2017; Derr et al., 2012; Driller-

80 Colangelo et al., 2016; Hariadi et al., 2015b; Furuta et al., 2013). Using this system, we

81 probed the role of motor-cargo linkers and found that the initiation of kinesin-3 ensemble

82 motility is dictated by the flexibility of the linkers. Thus, the DNA origami tool described

83 here becomes a robust system that can be extended to study regulatory mechanisms of

84 other molecular motors in their ensemble state. 


\section{Results}

\section{Design and validation of $6 \mathrm{HB}-400 \mathrm{~nm}$ DNA origami scaffold}

93 Molecular motors have been extensively studied at the single molecule level, however

94 while performing their cellular functions they often work in ensembles. Few studies have

95 created synthetic DNA scaffolds to study motor ensembles and so far, the systems that

96 exist can assemble maximum of 6 motors with regular spacing (Derr et al., 2012; Hariadi

97 et al., 2015b). To expand the capabilities in studying motor ensembles, here we adapted a

98 DNA origami scaffold that was designed earlier (Bui et al., 2010). The DNA origami

99 scaffold used in this study is 400nm long with 6 helix bundles (6HB-400nm) (Methods)

100 (Figure 1A \& Supplementary Figure 1). The 6HB-400nm scaffold has been designed to

101 accommodate up to 28 oligonucleotide-overhangs (called handles) in a linear stretch,

102 each handle is spaced $\sim 14 \mathrm{~nm}$ apart (Supplementary Figure 1). The 6HB-400nm scaffold

103 was validated to confirm its structure and occupancy of oligonucleotide handles using

104 anti-handles conjugated with fluorescent molecules and motors at helix 1

$105 \& 5$ and helix 2 respectively (Methods) (Supplementary Table $1 \&$ Supplementary Figure

106 2A-D). For the protein attachment to the $6 \mathrm{HB}-400 \mathrm{~nm}$ scaffold we designed two versions;

107 A 40 and 20 base single-stranded oligonucleotide (anti-handle) that is covalently linked

108 to the tail end of kinesin through SNAP-tag (Methods) (Supplementary Figure 3A \& 3B).

109 The 20 base oligonucleotides anti-handle is fully complementary to the handle sites

110 emanating from the 6HB-400nm scaffold mimicking the rigid linker (Supplementary

111 Figure $3 \mathrm{C}$ ). In the case of 40 base oligonucleotides anti-handle, the complementarity is

112 restricted only towards 20 basepairs at the 5 of the handle sites rendering them as

113 flexible linkers. On the other hand, the handle oligonucleotides that are complementary to

114 the anti-handles are also 40 base long of which 20 base towards the $3 \% 5$ end (i.e.,

115 towards the DNA scaffold) remain single-stranded (Supplementary Figure 3C). From

116 hereafter we refer the 20 and 40 oligonucleotides protein-DNA scaffold attachment

117 linkers as rigid and flexible linkers respectively (Supplementary Figure 3C).

119 Next, we established motor motility assays assessing the feasibility of the 6HB-400nm

120 scaffold to study the ensemble motor behavior. Briefly, KIF1A motors were covalently

121 linked to single-stranded DNA oligonucleotides (flexible and rigid anti-handles) that are 
complementary to the handles present in the 6HB-400nm scaffold (Methods) (Figure 1C

123

124

125

126

127

128

129

130

131

132

133

134

135

136

137

138

139

140

141

142

143

144

145

146

147

148

149

150

151

152

and Supplementary Figure 3A \& 3B). To visualize the 6HB-400nm scaffolds in our motility assays, we labelled them using six fluorescent oligonucleotides that were attached to the fluorescent handles at helix $1 \& 5$ (Methods) (Figure 1A, Supplementary Figure $1 \&$ Supplementary Table 1). In our motility assays, we compared the 6HB400nm:28 KIF1A motors (ensemble motors) motility against individual KIF1A motors (Methods). Our results show that the velocity of 6HB-400nm:28 KIF1A ensemble and single KIF1A motors are $1.27 \pm 0.39 \mu \mathrm{m} \mathrm{s}^{-1}$ and $1.08 \pm 0.29 \mu \mathrm{m} \mathrm{s}^{-1}$ respectively (Figure 1D $\& 1 \mathrm{E}$ ) (Supplementary Table 2). In the case of processivity, the 6HB-400nm:16 KIF1A ensembles and single KIF1A motors are near identical (Figure 1F) (Supplementary Table 1). From this experiment we conclude that the $6 \mathrm{HB}-400 \mathrm{~nm}$ scaffold can serve as a tool for characterizing motor ensemble properties.

\section{Motility properties of KIF1A motor ensembles}

Full-length Kinesin-3 motors can exist as monomeric units and homodimers in cells, single-molecule studies have suggested that the dimeric KIF1A motors are superprocessive (Soppina et al., 2014; Tomishige et al., 2002). However, a quantitative comparison of monomer versus dimer KIFA ensembles is yet to be characterized. Moreover, the flexibility of cargo-motor linkers in an ensemble setting has not been explored. In order to test this, we compared the ensemble motility properties of dimeric and monomeric KIF1A tethered to $6 \mathrm{HB}-400 \mathrm{~nm}$ scaffold, hereafter called as $6 \mathrm{HB}-400 \mathrm{~nm}$ monomer KIFA and dimer KIF1A ensembles respectively (Methods) (Supplementary Figure 3A). We chose four different ensemble numbers in this study; 28, 16, 8 and 4 motor attachment handle sites, which we hereafter refer to as $28 \mathrm{H}, 16 \mathrm{H}, 8 \mathrm{H}$ and $4 \mathrm{H}$ respectively (Methods). The 6HB-400nm:KIF1A dimer ensembles with varying handle sites and linkers were analyzed in an agarose gel to confirm the extent of DNA:motor complex formation (Methods) (Figure 1C, Supplementary Figure 3E \& 3F).

From the motility assays, we could record individual 6HB-400nm:KIF1A ensembles moving along the microtubules, regardless of the KIF1A oligomeric state, ensemble number and the nature of linkers. Upon quantification of velocity and processivity values, 
153

154

155

156

157

158

159

160

161

162

163

164

165

166

167

168

169

170

171

172

173

174

175

176

177

178

179

180

181

182

183

deviations between different 6HB-400nm:KIF1A ensembles began to emerge (Figure $2 \&$ Supplementary Figure $4 \& 5$ ). The velocities within monomer and dimer KIF1A ensemble cohorts does not change with motor number. However, the velocity between monomer and dimer KIF1A rigid ensembles are two-fold different; for 6HB-400nm: 4$28 \mathrm{H}$ dimer and monomer KIF1A rigid ensembles the average velocity is $\sim 2 \mu \mathrm{m} \mathrm{s}^{-1}$ and $\sim 0.9 \mu \mathrm{m} \mathrm{s}^{-1}$ respectively (Figure 2A, Supplementary Figure $4 \&$ Supplementary Table 2). Compared to the KIF1A monomer rigid ensembles the flexible cohort exhibited two-fold reduction in velocity (Figure 2A, Supplementary Figure $4 \&$ Supplementary Table 2). In contrast, the processivity values follow similar trend between monomer rigid, monomer flexible and dimer rigid ensembles (Figure 2B, Supplementary Figure 4 \& Supplementary Table 2). A puzzling phenomenon here is the poor processivity of KIF1A ensembles below $16 \mathrm{H}$. The $6 \mathrm{HB}-400 \mathrm{~nm}-4 \mathrm{H}$ and $8 \mathrm{H}$ KIF1A dimer processivity is $4.96 \pm 0.01 \mu \mathrm{m}$ and $4.95 \pm 0.01 \mu \mathrm{m}$ respectively. We attribute this low processivity of $6 \mathrm{HB}-400 \mathrm{~nm}-4 \mathrm{H} \& 8 \mathrm{H}$ KIF1A dimer ensembles to the high magnesium concentration used in our motility assays. High magnesium levels are required to overcome the magnesium quenching property of 6HB-400nm DNA scaffolds and therefore balancing the requirement of $\mathrm{Mg} 2+$ ions for motor ATPase activity (Methods). Indeed, when high magnesium is used in motility assays the single dimeric KIF1A also exhibits poor processivity of $3.46 \pm 0.01$ $\mu \mathrm{m}$ (Figure 1E \& Supplementary Table 2). We also observed that the high magnesium induced low processivity can be overcome by higher motor ensembles. For example, the processivity of $6 \mathrm{HB}-400 \mathrm{~nm}$ dimer KIF1A rigid $16 \mathrm{H}$ and $28 \mathrm{H}$ ensembles are $12.2 \pm 0.01$ $\mu \mathrm{m}$ and 7.2 $\pm 0.01 \mu \mathrm{m}$ respectively and is similar to the dimeric KIF1A processivity $9.8 \pm 0.01 \mu \mathrm{m}$ (Supplementary Table 2 ).

In summary, the velocity of KIF1A ensembles remains unaffected regardless of the motor number and the processivity of KIF1A ensembles improves as the motor number increases.

\section{Comparative analysis of flexible versus rigid cargo-motor linkers}

While the velocity and processivity of flexible versus rigid cargo-motor linkers in ensembles remain largely unchanged, we observed reduced number of $6 \mathrm{HB}-400 \mathrm{~nm}$ 
184 KIF1A monomer ensembles with flexible linkers encountering the microtubule

185 (Supplementary Movie 1). To gain more insights, we qualitatively assessed the ability of flexible and rigid linked 6HB-400nm KIF1A monomer ensembles binding microtubules

187 in the absence of ATP, a microtubule strong binding condition called rigor-state

188 (Methods). The rigor-state assays exemplify our observation that the 6HB-400nm KIF1A monomer ensembles with flexible linkers show reduced binding to the microtubules

190 (Figure 3). In order to systematically probe the cargo-motor linkers, we additionally generated linkers that have single-stranded stretch at the either end of scaffold or motor tail, called intermediate flexible linkers (Methods) (Supplementary Table 1). In the rigorstate assays the 6HB-400nm KIF1A monomer ensembles linked with intermediate

194 flexible linkers also showed a marked decrease in microtubule binding (Figure 3). The reduced binding of $6 \mathrm{HB}-400 \mathrm{~nm}$ KIF1A monomer ensembles is a recurring phenomenon regardless of motor number sampled in our assays (Figure $3 \&$ Supplementary Figure 6).

Next, we reasoned whether the reduced microtubule-binding ability of $6 \mathrm{HB}-400 \mathrm{~nm}$ KIF1A monomer ensembles with flexible linkers could be rescued. For which we designed rescue oligonucleotides that can basepair with the single-stranded stretches in the flexible linkers (Methods) (Figure 3, Supplementary Figure 6 \& Supplementary Table 1). The rigor-state assays were performed in the absence and presence of rescue oligonucleotides for flexible 6HB-400nm monomer KIF1A ensembles (Methods).

204 Remarkably, the microtubule-binding of 6HB-400nm KIF1A monomer ensembles with 205 the flexible linkers was restored upon the addition of rescue oligonucleotides (Figure 3). 206 The microtubule-binding rescue was observed with $16 \mathrm{H}$ also, however, the effects were 207 more pronounced with 8H (Figure 3 \& Supplementary Figure 6). To establish whether 208 the rescue is specific, we performed the rigor-state rescue experiments in the presence of 209 a scrambled oligonucleotides (Supplementary Table 1). In comparison to the rescue 210 oligonucleotides that have complementarity to the flexible linkers the scrambled 211 oligonucleotides did not show any improvement in microtubule-binding of 6HB-400nm

212 KIF1A monomer ensembles (Figure 3 \& Supplementary Figure 6). These experiments 213 conclusively demonstrate that the microtubule-binding of KIF1A monomer ensembles 214 can be regulated by the stiffness of the cargo-motor linker. 
216 Initiation of KIF1A ensemble motility is sensitive to the rigidity of DNA linkers

217 The above rescue experiments were performed in the rigor-state. To assess the functional

218 significance of cargo-motor linker flexibility, we next performed experiments in the

219 presence of ATP (Methods). In addition to the velocity and processivity values, we

220 measured the binding frequency of flexible 6HB-400nm KIF1A monomer ensembles

221 with and without the rescue oligonucleotides (Methods). The velocity and processivity

222 values of flexible 6HB-400nm KIF1A monomer ensembles do not change upon addition

223 of rescue oligonucleotides (Figure 4A, Supplementary Figure 7 \& Supplementary Table

224 1), similar to our results with flexible versus rigid linkers (Figure 2). However, we

225 observed a marked decrease in landing frequency with the flexible 6HB-400nm KIF1A

226 monomer ensembles (Figure 4B). The landing frequency rate of flexible 6HB-400nm 4H,

$2278 \mathrm{H}$ and 16H KIF1A ensembles are 0.004, 0.001 and $0.007 \mu^{-1} \mathrm{~s}^{-1}$ respectively. Similar

228 to the rigor-state experiments, we performed the motility experiments with flexible 6HB-

$229400 \mathrm{~nm}$ KIF1A monomer ensembles in the presence of rescue oligonucleotides

230 (Methods). The number of flexible 6HB-400nm KIF1A monomer ensemble particles

231 moving on the microtubules markedly increases with rescue oligonucleotides

232 (Supplementary Movie 1). Upon quantification, the landing-frequency of flexible 6HB-

$233400 \mathrm{~nm}$ KIF1A monomer ensembles significantly increases with the addition of rescue

234 oligonucleotides in the motility assays (Figure 4B \& Supplementary Figure 7). The

235 landing frequencies of rescued $6 \mathrm{HB}-400 \mathrm{~nm} 4 \mathrm{H}, 8 \mathrm{H}$ and $16 \mathrm{H}$ KIF1A monomer ensembles

236 are $0.011,0.073$ and $0.059 \mu^{-1} \mathrm{~s}^{-1}$ respectively. We also observed the improvement in

237 landing frequency is specific to the rescue oligonucleotides that are complimentary to the

238 single-stranded DNA linkers. Combinedly these experiments suggest that the initiation of

239 KIF1A ensembles motility is sensitive to the rigidity of the linkers between the

2406 HB400nm scaffold and motors. 


\section{Discussion}

247 Majority of the motor regulation studies have been performed using purified components

248 of single or individual motor proteins. There are a few exceptions where the tug-of-war

249 between two opposing motors have been described (Derr et al., 2012; Hariadi et al.,

250 2015b; Toropova et al., 2017; Driller-Colangelo et al., 2016; Furuta et al., 2013).

251 However, the regulatory aspects of ensemble motors have been poorly described, for

252 instance the effects of cargo-motor linker flexibility towards ensemble kinesin-3 motility

253 is unknown. Similarly, the ensemble motor experiments so far has been limited to DNA

254 origami scaffolds with six motors. On the other hand, DNA scaffolds that can

255 accommodate hundreds of motors has been described. However, it can only control the

256 spacing between each motor thus limiting the ability to control the motor ensemble

257 numbers (Hariadi et al., 2015a). Molecular motors that work together have their ensemble

258 number in varying orders. For example, the motor ensemble numbers involved in the

259 intracellular and intraflagellar cargo transport can range from 2 - 20 (Hirokawa et al.,

260 2009b; Rai et al., 2016; Siddiqui and Straube, 2017; Prevo et al., 2017), and in the

261 sarcomere more than 50 muscle myosins can engage during muscle contraction (Spudich,

262 2014). In order to expand the capabilities to understand the molecular motor ensembles in

263 this study we have designed and validated a 6HB-400nm DNA origami scaffold. The

264 6HB-400nm DNA origami scaffold also acts as a cargo mimic, where the cargo-motor

265 linkers are amenable for varying degrees of flexibility. Therefore, the 6HB-400nm DNA

266 origami scaffold described here offers versatility to study molecular motor ensembles up

267 to 28 molecules.

Using 6HB-400nm DNA origami scaffold as a cargo, we tethered them with the KIF1A motors with varying ensemble motor numbers and linker flexibilities. From our assays

271 we show that the biochemical properties of the KIF1A motors does not get affected as

272 evident from the velocity and processivity values between rigid and flexible linkers.

273 However, a striking observation emerged from these assays is the inability of KIF1A

274 monomer ensembles to initiate motility when tethered to $6 \mathrm{HB}-400 \mathrm{~nm}$ with flexible

275 linkers (Figure 3 and 4). We also unequivocally show that the effects of flexible linkers

276 can be reversed by the addition of oligonucleotides that are complementary to the flexible 
277 single-strand DNA linkers i.e., the flexible linkers (Figure 3 and 4). We attribute the

278 mechanism of diminished microtubule-binding of KIF1A ensembles with flexible linkers

279 to its autoinhibited state mediated by the K-loop. In the case where the $6 \mathrm{HB}-400 \mathrm{~nm}$

280 KIF1A monomer ensembles tethered with flexible oligonucleotides, the motor domain

281 can adopt several degrees of conformation with respect to the $6 \mathrm{HB}-400 \mathrm{~nm}$ scaffold. This

282 might be conducive for an electrostatic interaction between the negatively charged DNA

283 strands of 6HB-400nm and the positively charged K-loop of KIF1A motor domain. Upon

284 addition of the rescue oligonucleotides complementary to the flexible regions the

persistence of the linkers decreases. Thus, the motor domain may remain with a restricted degree of conformational flexibility and no electrostatic interaction between K-loop and 6HB-400nm DNA elements.

The K-loop of kineins-3 motors is a unique element that has been shown to enhance the processivity among this subfamily of kinesin motors (Soppina and Verhey, 2014). This is mediated by the electrostatic interaction with the acidic carboxy-terminal tails of alphaand beta-tubulins. Therefore, it is conceivable that such an electrostatic interaction between K-loop and other acidic elements might sterically interfere with microtubule interaction. However, such an involvement of K-loop towards autoinhibitory regulation of kinesin-3 motors has so far not been described. Indeed, the autoinhibitory effects observed between the K-loop of KIF1A and DNA elements of 6HB-400nm scaffold are of non-physiological nature. However, a stretch of acidic amino acids is present in the coiled-coil regions of kinesin-3 motors that can mediate such an electrostatic interaction. Additionally, the MAP mediated regulation of kinesin-3 family motors might also involve such an electrostatic interaction (Monroy et al., 2020). Dimerization of kinesin-3 motors has been proposed to be an important regulatory step for their motility (Soppina et al., 2014; Al-Bassam et al., 2003; Patel et al., 2021; Siddiqui and Straube, 2017;

303 Hammond et al., 2009). Since multimerization of kinesin-3 monomers can also lead to 304 intracellular cargo transport (Schimert et al., 2019), here we propose an additional tier of 305 autoinhibitory regulation of kinesin-3 motors mediated by the K-loop. Autoinhibition of 306 motors by the cargo domain is a common feature among kinesin family motors (Sweeney and Holzbaur, 2018). In this study using DNA origami and ensemble motors we have 
illuminated an autoinhibition mechanism that was not described previously. Further underscoring the power of studying motors in ensembles and the importance of the 6HB400nm DNA origami scaffold developed in this study.

\section{Materials and Methods}

\section{Protein Purification}

314 Truncated Rat KIF1A (1-393 amino acids, dimer) followed by a GCN4 leucine zipper 315 and Rat KIF1A (1-369 amino acids, monomer) was cloned into a pET-17b vector with a 316 SNAP-tag followed by a 10X Histidine-tag at the carboxy-terminus. All the KIF1A gene 317 constructs were expressed using the BL21(DE3) bacterial expression system.

318 Transformed cells were grown at $37^{\circ} \mathrm{C}$ to $0 . \mathrm{D}$ 0.4-0.6 followed by induction with $0.5 \mathrm{mM}$ 319 IPTG and overnight shaking at $18^{\circ} \mathrm{C}$. Cells were harvested and lysed in Buffer-A 320 containing $25 \mathrm{mM}$ pipes, $\mathrm{pH} 6.8,100 \mathrm{mM} \mathrm{KCl}, 5 \mathrm{mM} \mathrm{MgCl} 2,5 \mathrm{mM} \beta$-mercaptoethanol, $32130 \mathrm{mM}$ imidazole. The supernatant was loaded onto a Ni-NTA column, followed by a high salt wash (Buffer-A with $300 \mathrm{mM} \mathrm{KCl}, 200 \mu \mathrm{M} \mathrm{ATP}$ ) followed by elution using Buffer-A with $350 \mathrm{mM}$ imidazole. Pure proteins were obtained by further subjecting the Ni-NTA elute to size exclusion chromatography using an S200 16/1600 column (GE) with Buffer-A.

\section{Preparation of Benzyl Guanine-labeled oligo}

328 Benzyl-guanine NHS ester (BG-GLA-NHS; NEB S2040) was covalently linked to the C7-amine modified oligonucleotide (Sigma), LPAH2 (see Supplementary Table 1). Briefly, 2mM LPAH2 (purchased from Sigma), was mixed with 20mM BG-NHS (dissolved in DMSO) in a molar ratio of 1:30 in the presence of 65mM HEPES pH 8.6 and incubated at $37^{\circ} \mathrm{C}$ with constant stirring overnight. The mixture was subjected to speed vacuum to get rid of the DMSO and further reconstituted in water. BG-labelled oligo was separated from excess BG-NHS and unlabeled oligo by subjecting this mixture to reverse phase HPLC using C18 column. Briefly, 100uL of the oligonucleotide mixture was injected into a clarity $5 \mathrm{u}$ oligo RP column (Phenomenex 00B-4442-E0) and was subjected to an increasing gradient of acetonitrile starting from $5 \%$ to $35 \%$ in $0.5 \mathrm{M}$ 
this elution. Labeling was confirmed by mass spectrometric analysis. The BG-labeled oligonucleotide peak was collected, speed vacuumed, and further purified using obtained

341 ethanol precipitation.

\section{Labelling Kinesin with Benzyl-Guanine oligonucleotide (BG-oligonucleotide)}

344 SNAP-tagged KIF1A proteins were incubated with a $2 \mathrm{X}$ molar excess of BG-labeled

345 oligonucleotide at room temperature for 15 minutes followed by overnight incubation at

$3464^{\circ} \mathrm{C}$. The removal of excess BG- oligonucleotide from the reaction was achieved by

347 subjecting the motor to Microtubule-binding and its release in the presence of ATP.

348 Active BG-labeled oligonucleotide motors obtained were flash frozen as 5uL aliquots and

349 stored at $-80^{\circ} \mathrm{C}$. In case of dimeric kinesin, excess BG-oligo removal was achieved by 350 another round of Ni-NTA purification. Labeling efficiency was assessed by a 10\% SDS-

351 PAGE gel as labeled protein showed a distinct gel shift (Supplementary Figure 3).

6HB-400nm DNA origami structure preparation

354 The 6HB-400nm structure was modified from a previously published structure (Bui et al., 355 2010). The sequences of all staple strands, and anti-handle stands are given in the 356 Supplementary Table 1. 10nM m13mp18 ssDNA (NEB S4040) were mixed with 100nM 357 core staples and 500nM handle staples to a total volume of 50uL in $1 \mathrm{X}$ folding buffer 358 (40mM Tris, 20mM acetic acid, 1mM EDTA, 12.5mM MgCl2, $\mathrm{pH} 8.0$ ), followed by 359 annealing in a PCR machine as follows: $90^{\circ} \mathrm{C}$ for 10 minutes, then cooled to $65^{\circ} \mathrm{C}$ with $36011^{\circ} \mathrm{C}$ per minute, then further cooled to $10^{\circ} \mathrm{C}$ with $1{ }^{\circ} \mathrm{C}$ per minute. The folded $6 \mathrm{HB}$ were 361 purified from excess staples using Amicon centriprep column.

\section{Motility and rigor assays}

364 Typically, a 5uL aliquot of 6HB-400nm ( 2nM) was mixed with $2 \mathrm{uL}$ of BG-labeled 365 oligonucleotide kinesin $(\sim 1 \mathrm{uM})$ and incubated for 1 hour at RT and subsequently in ice 366 for the duration of the assay. Successful formation of motor-6HB complex was assessed 367 by $1 \%$ agarose gel where complexes showed graded retardation in mobility with 368 increasing number of motors in the complex (Figure 1 and Supplementary Figure 3). 
using biotin-streptavidin chemistry. $0.9 \mathrm{uL}$ of motility mixture (motor-6HB-400nm

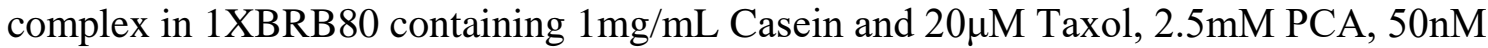
PCD, 50nM Trolox and 2mM ATP) was added to the motor-6HB-400nm complex and flowed onto the motility chamber containing microtubules. In the case of rigor experiments, ATP was omitted from the motility mixture. For rescue experiments, respective oligonucleotides as indicated was added to the motor:6HB-400nm mixture, for detailed information regarding the sequence of oligonucleotides see Supplementary Table1. Single-molecule motility of DNA-motor complex was imaged using a Nikon Ti-2 microscope (1.49 N.A., 100x objective) using total internal reflection microscopy (TIRF); images were acquired with a Hamamatsu sCMOS camera and NIS Elements software at $1-5 \mathrm{~Hz}$ intervals. Velocities were calculated using kymograph analysis in ImageJ software and Fiesta software.

References

Al-Bassam, J., Y. Cui, D. Klopfenstein, B.O. Carragher, R.D. Vale, and R.A. Milligan. 2003. Distinct conformations of the kinesin Unc104 neck regulate a monomer to dimer motor transition. J. Cell Biol. 163:743-753. doi:10.1083/jcb.200308020.

Bui, H., C. Onodera, C. Kidwell, Y. Tan, E. Graugnard, W. Kuang, J. Lee, W.B. Knowlton, B. Yurke, and W.L. Hughes. 2010. Programmable Periodicity of Quantum Dot Arrays with DNA Origami Nanotubes. Nano Lett. 10:3367-3372. doi:10.1021/nl101079u.

Derr, N.D., B.S. Goodman, R. Jungmann, A.E. Leschziner, W.M. Shih, and S.L. ReckPeterson. 2012. Tug-of-war in motor protein ensembles revealed with a programmable DNA origami scaffold. Science. 338:662-665. doi:10.1126/science.1226734.

Driller-Colangelo, A.R., K.W.L. Chau, J.M. Morgan, and N.D. Derr. 2016. Cargo rigidity affects the sensitivity of dynein ensembles to individual motor pausing. Cytoskelet. Hoboken NJ. 73:693-702. doi:10.1002/cm.21339. Measuring collective transport by defined numbers of processive and nonprocessive kinesin motors. Proc. Natl. Acad. Sci. U. S. A. 110:501-506. doi:10.1073/pnas.1201390110.

404

Gennerich, A., and R.D. Vale. 2009. Walking the walk: how kinesin and dynein coordinate their steps. Curr. Opin. Cell Biol. 21:59-67. doi:10.1016/j.ceb.2008.12.002. 
Hammond, J.W., T.L. Blasius, V. Soppina, D. Cai, and K.J. Verhey. 2010. Autoinhibition of the kinesin-2 motor KIF17 via dual intramolecular mechanisms. J. Cell Biol. 189:1013-1025. doi:10.1083/jcb.201001057.

Hammond, J.W., D. Cai, T.L. Blasius, Z. Li, Y. Jiang, G.T. Jih, E. Meyhofer, and K.J. Verhey. 2009. Mammalian Kinesin-3 motors are dimeric in vivo and move by processive motility upon release of autoinhibition. PLoS Biol. 7:e72. doi:10.1371/journal.pbio.1000072.

Hariadi, R.F., R.F. Sommese, A.S. Adhikari, R.E. Taylor, S. Sutton, J.A. Spudich, and S. Sivaramakrishnan. 2015a. Mechanical coordination in motor ensembles revealed using engineered artificial myosin filaments. Nat. Nanotechnol. 10:696-700. doi:10.1038/nnano.2015.132.

Hariadi, R.F., R.F. Sommese, and S. Sivaramakrishnan. 2015b. Tuning myosin-driven sorting on cellular actin networks. eLife. 4. doi:10.7554/eLife.05472.

Hirokawa, N., R. Nitta, and Y. Okada. 2009a. The mechanisms of kinesin motor motility: lessons from the monomeric motor KIF1A. Nat. Rev. Mol. Cell Biol. 10:877-884. doi: $10.1038 / \mathrm{nrm} 2807$.

Hirokawa, N., Y. Noda, Y. Tanaka, and S. Niwa. 2009b. Kinesin superfamily motor proteins and intracellular transport. Nat. Rev. Mol. Cell Biol. 10:682-696. doi:10.1038/nrm2774.

Huo, L., Y. Yue, J. Ren, J. Yu, J. Liu, Y. Yu, F. Ye, T. Xu, M. Zhang, and W. Feng. 2012. The CC1-FHA tandem as a central hub for controlling the dimerization and activation of kinesin-3 KIF1A. Struct. Lond. Engl. 1993. 20:1550-1561. doi:10.1016/j.str.2012.07.002.

Imanishi, M., N.F. Endres, A. Gennerich, and R.D. Vale. 2006. Autoinhibition regulates the motility of the C. elegans intraflagellar transport motor OSM-3. J. Cell Biol. 174:931-937. doi:10.1083/jcb.200605179.

Lessard, D.V., O.J. Zinder, T. Hotta, K.J. Verhey, R. Ohi, and C.L. Berger. 2019. Polyglutamylation of tubulin's C-terminal tail controls pausing and motility of kinesin-3 family member KIF1A. J. Biol. Chem. 294:6353-6363. doi:10.1074/jbc.RA118.005765.

McKenney, R.J., W. Huynh, R.D. Vale, and M. Sirajuddin. 2016. Tyrosination of $\alpha-$ tubulin controls the initiation of processive dynein-dynactin motility. EMBO J. 35:1175-1185. doi:10.15252/embj.201593071.

Monroy, B.Y., T.C. Tan, J.M. Oclaman, J.S. Han, S. Simó, S. Niwa, D.W. Nowakowski, R.J. McKenney, and K.M. Ori-McKenney. 2020. A Combinatorial MAP Code Dictates Polarized Microtubule Transport. Dev. Cell. 53:60-72.e4. doi:10.1016/j.devcel.2020.01.029. 
Okada, Y., and N. Hirokawa. 1999. A processive single-headed motor: kinesin superfamily protein KIF1A. Science. 283:1152-1157. doi:10.1126/science.283.5405.1152.

Patel, N.M., M.S.A. Siva, R. Kumari, D.J. Shewale, A. Rai, M. Ritt, P. Sharma, S.R.G. Setty, S. Sivaramakrishnan, and V. Soppina. 2021. KIF13A motors are regulated by Rab22A to function as weak dimers inside the cell. Sci. Adv. 7:eabd2054. doi:10.1126/sciadv.abd2054.

Prevo, B., J.M. Scholey, and E.J.G. Peterman. 2017. Intraflagellar transport: mechanisms of motor action, cooperation, and cargo delivery. FEBS J. 284:2905-2931. doi:10.1111/febs.14068.

Rai, A., D. Pathak, S. Thakur, S. Singh, A.K. Dubey, and R. Mallik. 2016. Dynein Clusters into Lipid Microdomains on Phagosomes to Drive Rapid Transport toward Lysosomes. Cell. 164:722-734. doi:10.1016/j.cell.2015.12.054.

Ren, J., S. Wang, H. Chen, W. Wang, L. Huo, and W. Feng. 2018. Coiled-coil 1mediated fastening of the neck and motor domains for kinesin-3 autoinhibition. Proc. Natl. Acad. Sci. U. S. A. 115:E11933-E11942. doi:10.1073/pnas.1811209115.

Scarabelli, G., V. Soppina, X.-Q. Yao, J. Atherton, C.A. Moores, K.J. Verhey, and B.J. Grant. 2015. Mapping the Processivity Determinants of the Kinesin-3 Motor Domain. Biophys. J. 109:1537-1540. doi:10.1016/j.bpj.2015.08.027.

Schimert, K.I., B.G. Budaitis, D.N. Reinemann, M.J. Lang, and K.J. Verhey. 2019. Intracellular cargo transport by single-headed kinesin motors. Proc. Natl. Acad. Sci. U. S. A. 116:6152-6161. doi:10.1073/pnas.1817924116.

Siddiqui, N., and A. Straube. 2017. Intracellular cargo transport by kinesin-3 motors. Biochem. Mosc. 82:803-815. doi:10.1134/S0006297917070057.

Sirajuddin, M., L.M. Rice, and R.D. Vale. 2014. Regulation of microtubule motors by tubulin isotypes and post-translational modifications. Nat. Cell Biol. 16:335-344. doi: $10.1038 / \mathrm{ncb} 2920$.

Soppina, V., S.R. Norris, A.S. Dizaji, M. Kortus, S. Veatch, M. Peckham, and K.J. Verhey. 2014. Dimerization of mammalian kinesin-3 motors results in superprocessive motion. Proc. Natl. Acad. Sci. U. S. A. 111:5562-5567. doi:10.1073/pnas.1400759111.

Soppina, V., and K.J. Verhey. 2014. The family-specific K-loop influences the microtubule on-rate but not the superprocessivity of kinesin-3 motors. Mol. Biol. Cell. 25:2161-2170. doi:10.1091/mbc.E14-01-0696. 
477 Spudich, J.A. 2014. Hypertrophic and dilated cardiomyopathy: four decades of basic research on muscle lead to potential therapeutic approaches to these devastating genetic diseases. Biophys. J. 106:1236-1249. doi:10.1016/j.bpj.2014.02.011.

480 Sweeney, H.L., and E.L.F. Holzbaur. 2018. Motor Proteins. Cold Spring Harb. Perspect. Biol. 10:a021931. doi:10.1101/cshperspect.a021931. kinesin into a processive motor after dimerization. Science. 297:2263-2267. doi:10.1126/science.1073386.

Toropova, K., M. Mladenov, and A.J. Roberts. 2017. Intraflagellar transport dynein is autoinhibited by trapping of its mechanical and track-binding elements. Nat. 
Figure Legends

Figure 1: Characterization of 400nm-6HB DNA origami as motor cargo scaffold. A.

491 Cartoon representation of 6HB-400nm DNA origami scaffold used in this study as

492 illustrated. 28 handle sites are located in the helix 2 as a single file and three fluorescent

493 handles are located each in helix 1 and 5. B. Negative stained electron micrograph of

494 6HB-400nm DNA origami scaffold. Scale bar $=50 \mathrm{~nm}$. C. Agarose gel shift assays for

495 6HB-400nm scaffold with and without KIF1A dimers for varying handle sites as marked.

496 D. Representative kymographs of KIF1A 1-393 SNAP-647 (grey) and 6HB-400nm 28H

497 KIF1A 1-393 SNAP ensemble motors (blue) moving on microtubules. Scale bar $=5 \mu \mathrm{m}$

498 and 10 seconds. E. Velocity and processivity histograms of KIF1A 1-393 SNAP-647 (in

499 grey) and 6HB-400nm 16H KIF1A 1-393 SNAP ensemble (in blue) motors. The average

500 velocity of KIF1A 1-393 SNAP-647 are $1.27 \pm 0.39 \mu \mathrm{m} \mathrm{s}^{-1}, \mathrm{n}=1006 \mathrm{HB}-400 \mathrm{~nm} \mathrm{16H}$

501 KIF1A 1-393 SNAP ensembles are $1.08 \pm 0.29 \mu \mathrm{m} \mathrm{s}^{-1}, \mathrm{n}=91$. The average run length of

502 KIF1A 1-393 SNAP-647 are 3.36 $\mu \mathrm{m}$ and 6HB-400nm 16H KIF1A 1-393 SNAP

503 ensembles are $9.8 \mu \mathrm{m}$. $\mathrm{n}$ represents the number of motor particles analyzed.

Figure 2: KIF1A dimer versus monomer ensemble motility. A. Average velocity data of 6HB-400nm 4-28H KIF1A monomer ensembles with flexible and rigid

507 oligonucleotides and KIF1A dimer ensembles with rigid oligonucleotides. B. Average

508 processivity values of $6 \mathrm{HB}-400 \mathrm{~nm} 4-28 \mathrm{H}$ KIF1A ensembles as described in A. Error bars

509 represent the standard error of the mean from three independent experiments. For

510 individual values see Supplementary Table 2.

512 Figure 3: Microtubule-binding of KIF1A ensembles with varying linkers.

513 Microtubule-binding of 6HB-400nm 8H KIF1A monomer ensembles with varying

514 linkers as indicated and illustrated. The microtubules are shown in magenta and the 6HB-

$515400 \mathrm{~nm}$ KIF1A 8H in cyan. In each microtubule-binding experiment, a rescue reaction

516 was perfumed (marked as rescue), where an oligonucleotide that is complementary to the

517 flexible parts of linkers was added to the mixture. For more details regarding the

518 sequences of linkers and rescue oligonucleotides see Supplementary Table 1. Scale bar = $5195 \mu \mathrm{m}$. 
Figure 4: Motility properties of KIF1A ensembles with varying linkers. A.

flexible and flexible-rescue oligonucleotides. Scale bar $=5 \mu \mathrm{m}$ and 10 seconds. $\mathbf{B}$. Average landing frequency rates for $6 \mathrm{HB}-400 \mathrm{~nm} 4 \mathrm{H}, 8 \mathrm{H}$ and $16 \mathrm{H} \mathrm{KIF1A}$ monomer ensembles with flexible linkers and rescue oligonucleotides as indicated. Error bars represent the standard error of the mean from three independent experiments.

fluorescent handle sites. A. Arrangement of m13mp18 single-stranded DNA as 6HB400nm DNA origami scaffold. The helices and corresponding base number are as indicated. B \& C. Zoomed in view of two distal end of 6HB-400nm DNA origami scaffold as color coded in A. The m13mp18 strand and the complementary staple oligos are marked as wavy lines in light and dark grey respectively. The fluorescent handle extensions are indicated as red arrows. The helices, column number and handle numbers $(\mathrm{H} 1, \mathrm{H} 2, \mathrm{H} 3 \ldots \mathrm{H} 26, \mathrm{H} 27 \& \mathrm{H} 28)$ and nucleotide base resolution are as indicated.

A. Agarose gel of m13mp18 scaffold (pre-folding lane), folded reaction of 400nm-6HB (post-folding lane) and 6HB-400nm after removing the excess oligonucleotides (postpurification lane). The is marked as $6 \mathrm{hb}$ and the size of ladder as indicated. B. Agarose

541 gel of 6HB-400nm with varying handle numbers incubated Cy3-labelled anti-handle

542 oligonucleotides as indicated, and the same gel was imaged under Cy3 and SYBR (UV)

543 channel. C. Agarose gel of 6HB-400nm with varying handle numbers incubated Cy3-

544 labelled anti-handle oligonucleotides as indicated. To rule out effects of the SYBR green,

545 the experiments were performed without SYBR. The same gel was imaged under Cy3

546 and SYBR (UV) channel. D. Mean fold increase in Cy3 fluorescence as a function of

547 handle number present in the 6HB-400nm DNA origami scaffold. Error bars represent

548 the standard error of the mean from three independent experiments. 
Supplementary Figure 3: Engineering kinesins for DNA attachment. A. Cartoon representation of KIF1A motors tagged with oligonucleotides using benzyl-guanine and SNAP tag method. B. SDS-PAGE gel of KIF1A monomer and dimer before and after tagged with flexible and rigid anti-handle oligonucleotides as indicated. The white, red and yellow asterisks denote KIF1A unconjugated, KIF1A conjugated with rigid and flexible oligonucleotides respectively. C. Illustration of 6HB-400nm DNA origami scaffold with flexible, rigid and intermediate motor linkers as indicated. For details regarding the sequences of oligonucleotides see Supplementary Table 1. D. Agarose gel shift assays for 6HB-400nm scaffold with and without KIF1A monomer ensembles for the $8 \mathrm{H}$ used in Figure 3 rigor experiments. E. \& F. Agarose gel shift assays for 6HB$400 \mathrm{~nm}$ scaffold with and without KIF1A monomers with varying handle sites for flexible and rigid linkers as marked.

Supplementary Figure 4: Kymographs of KIF1A ensemble motility. Representative kymographs of KIF1A ensembles with flexible and rigid anti-handle oligonucleotides, for the data shown in Figure 2 as indicated. Scale bar $=5 \mu \mathrm{m}$ and 10 seconds. motility. Velocity and processivity histograms of 6HB-400nm 4-28H KIF1A monomer and dimer ensembles. Data for KIF1A monomer ensembles with flexible, rigid and KIF1A dimer ensembles with rigid anti-handle oligonucleotides are shown as indicated.

\section{Supplementary Figure 6: Microtubule binding of KIF1A 16H ensembles.}

573 Microtubule-binding of 6HB-400nm 16H KIF1A monomer ensembles with varying

574 linkers as indicated and illustrated. The microtubules are shown in magenta and the 6HB-

$575400 \mathrm{~nm}$ KIF1A ensembles in cyan. In each microtubule-binding experiment, a rescue

576 reaction was perfumed (marked as rescue), where an oligonucleotide that is

577 complimentary to the flexible parts of linkers was added to the mixture. For more details

578 regarding the sequences of linkers and rescue oligonucleotides see Supplementary Table

579 1. Scale bar $=5 \mu \mathrm{m}$. 
581 Supplementary Figure 7: Histograms and kymographs of KIF1A ensembles with

582 flexible and rescue linkers. A. Velocity and processivity histograms of 6HB-400nm 4-

583 28H KIF1A monomer ensembles with flexible and flexible-rescue oligonucleotides, for

584 the data shown in Figure 4.

585

586 Supplementary Movie 1: KIF1A 8H ensembles with rigid, flexible and flexible with

587 rescue oligonucleotides as indicated. Scale bar $=5 \mu \mathrm{m}$ and time in minutes $/ \mathrm{seconds}$

588 (mm:ss format) as indicated.

589

590

591 


\section{$592 \quad$ Acknowledgments}

593 We thank the Sirajuddin lab members for critical feedback on the manuscript. P.L

594 acknowledges the support from inStem Graduate Program. M.S acknowledges funding

595 support from inStem core grants from the Department of Biotechnology, India, a

596 DBT/Wellcome Trust India Alliance Intermediate Fellowship (IA/I/14/2/501533), EMBO

597 Young Investigator Programme award, CEFIPRA (5703-1), the Department of Science

598 and Technology, SERB-EMR grant (CRG/2019/003246) and DBT-BIRAC

599 (BT/PR40389/COT/142/6/2020).

600 Author Contribution

601 P.L and M.S designed the work. P.L performed the experiments and analyzed the data.

602 M.S supervised the work and wrote the paper.

603

604 Competing interests

605 The authors declare no conflict of interest. 
bioRxiv preprint doi: https://doi.org/10.1101/2021.12.02.470904; this version posted December 2, 2021. The copyright holder for this preprint (which was not certified by peer review) is the author/funder, who has granted bioRxiv a license to display the preprint in perpetuity. It is made

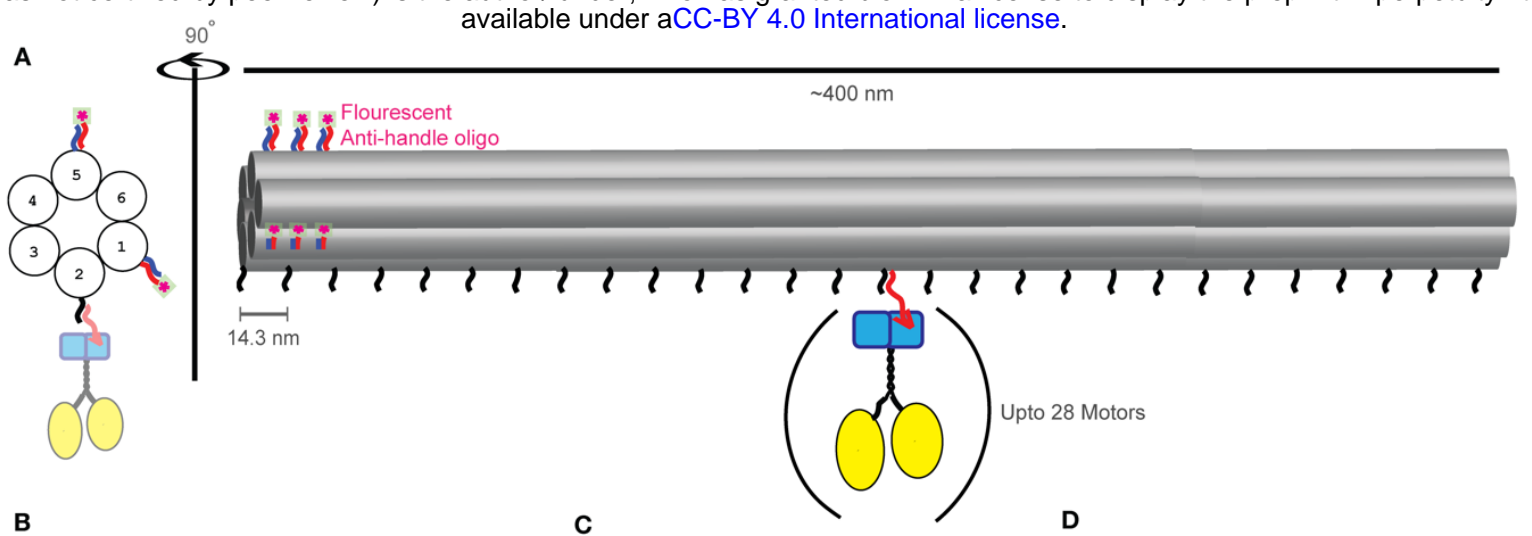

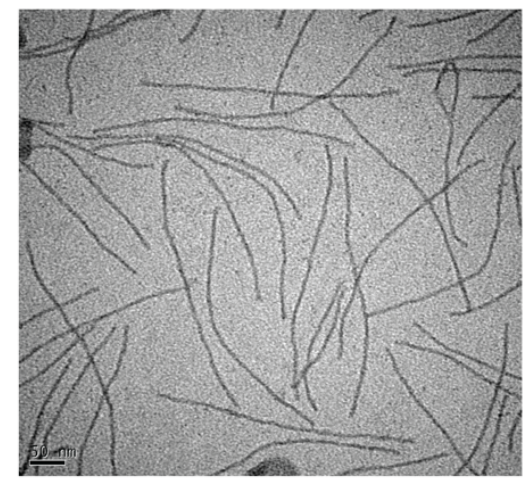

E

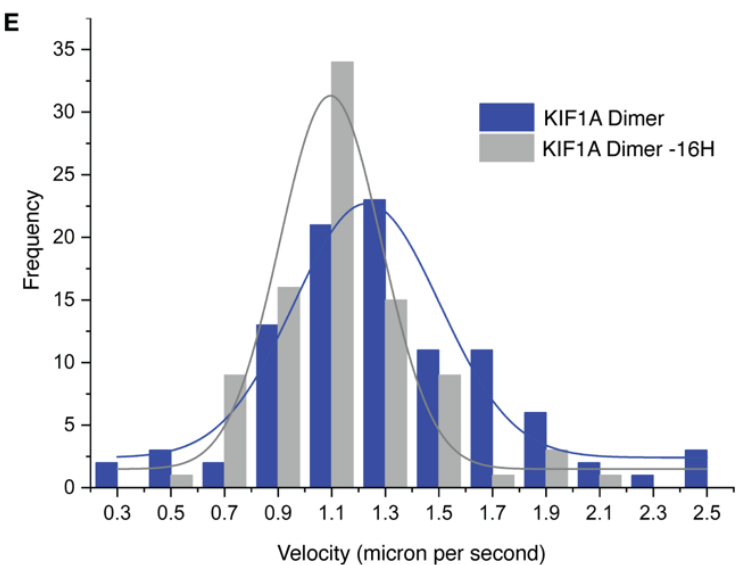

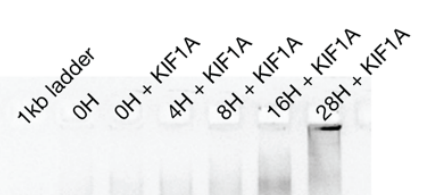
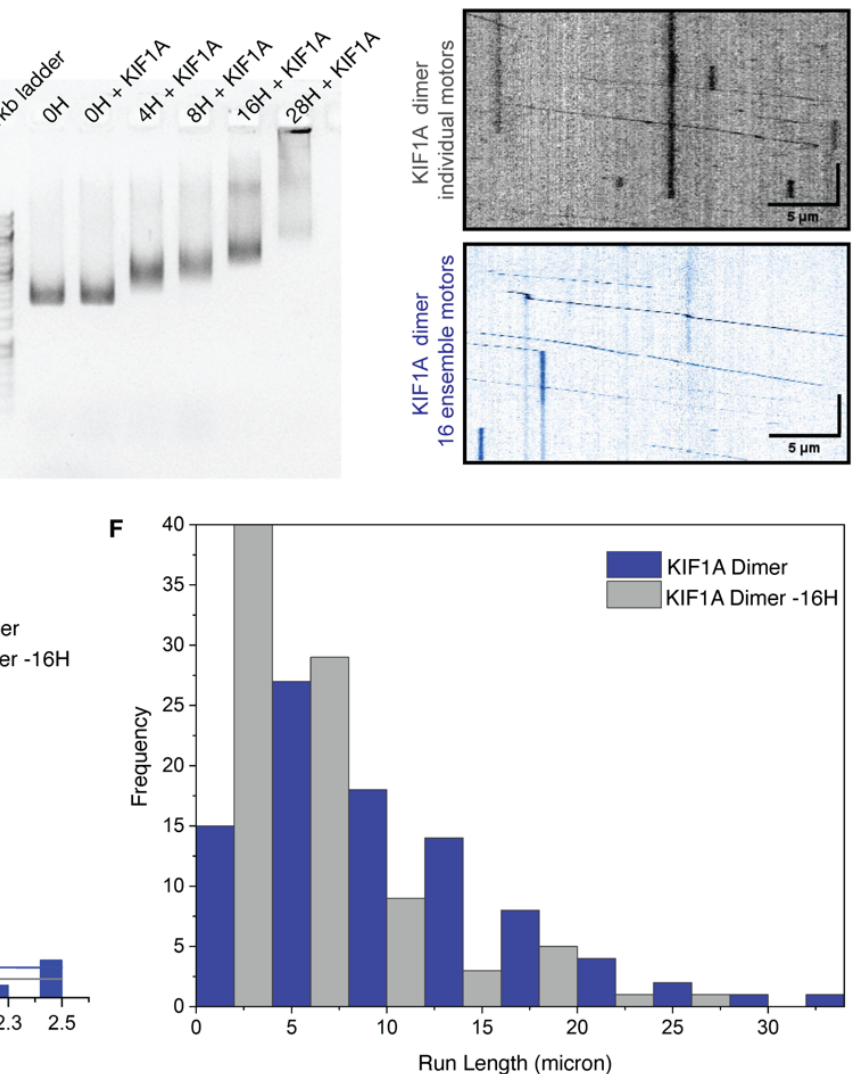

Figure 1: Characterization of 400nm-6HB DNA origami as motor cargo scaffold.

A. Cartoon representation of $6 \mathrm{HB}-400 \mathrm{~nm}$ DNA origami scaffold used in this study as illustrated. 28 handle sites are located in the helix 2 as a single file and three fluorescent handles are located each in helix 1 and 5. B. Negative stained electron micrograph of $6 \mathrm{HB}-400 \mathrm{~nm}$ DNA origami scaffold. Scale bar $=50 \mathrm{~nm}$. C. Agarose gel shift assays for $6 \mathrm{HB}-400 \mathrm{~nm}$ scaffold with and without KIF1A dimers for varying handle sites as marked. D. Representative kymographs of KIF1A 1-393 SNAP-647 (grey) and 6HB-400nm 28H KIF1A 1-393 SNAP ensemble motors (blue) moving on microtubules. Scale bar $=5 \mu \mathrm{m}$ and 10 seconds. E. Velocity and processivity histograms of KIF1A 1-393 SNAP-647 (in grey) and 6HB-400nm 16H KIF1A 1-393 SNAP ensemble (in blue) motors. The average velocity of KIF1A 1-393 SNAP-647 are $1.27 \pm 0.39 \mu \mathrm{m} \mathrm{s}^{-1}, \mathrm{n}=1006 \mathrm{HB}-400 \mathrm{~nm} 16 \mathrm{H}$ KIF1A 1-393 SNAP ensembles are $1.08 \pm 0.29 \mu \mathrm{m} \mathrm{s}^{-1}, \mathrm{n}=91$. The average run length of KIF1A 1-393 SNAP-647 are $3.36 \mu \mathrm{m}$ and $6 \mathrm{HB}-400 \mathrm{~nm} 16 \mathrm{H}$ KIF1A 1-393 SNAP ensembles are $9.8 \mu \mathrm{m} . \mathrm{n}$ represents the number of motor particles analyzed. 
bioRxiv preprint doi: https://doi.org/10.1101/2021.12.02.470904; this version posted December 2, 2021. The copyright holder for this preprint (which was not certified by peer review) is the author/funder, who has granted bioRxiv a license to display the preprint in perpetuity. It is made available under aCC-BY 4.0 International license.

A

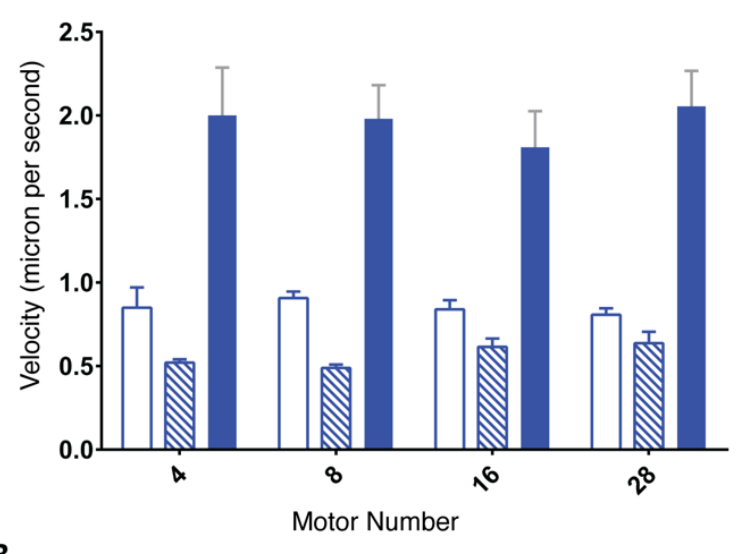

B

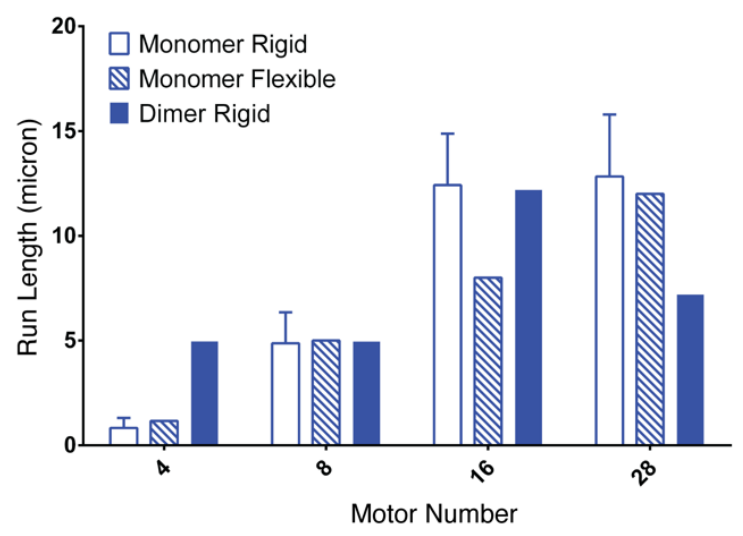

Figure 2: KIF1A dimer versus monomer ensemble motility. A. Average velocity data of $6 \mathrm{HB}-400 \mathrm{~nm} 4-28 \mathrm{H}$ KIF1A monomer ensembles with flexible and rigid oligonucleotides and KIF1A dimer ensembles with rigid oligonucleotides. B. Average processivity values of $6 \mathrm{HB}-400 \mathrm{~nm}$ 4-28H KIF1A ensembles as described in A. Error bars represent the standard error of the mean from three independent experiments. For individual values see Supplementary Table 2. 


\section{Lama and Sirajuddin 2021}

bioRxiv preprint doi: https://doi.org/10.1101/2021.12.02.470904; this version posted December 2, 2021. The copyright holder for this preprint (which was not certified by peer review) is the author/funder, who has granted bioRxiv a license to display the preprint in perpetuity. It is made

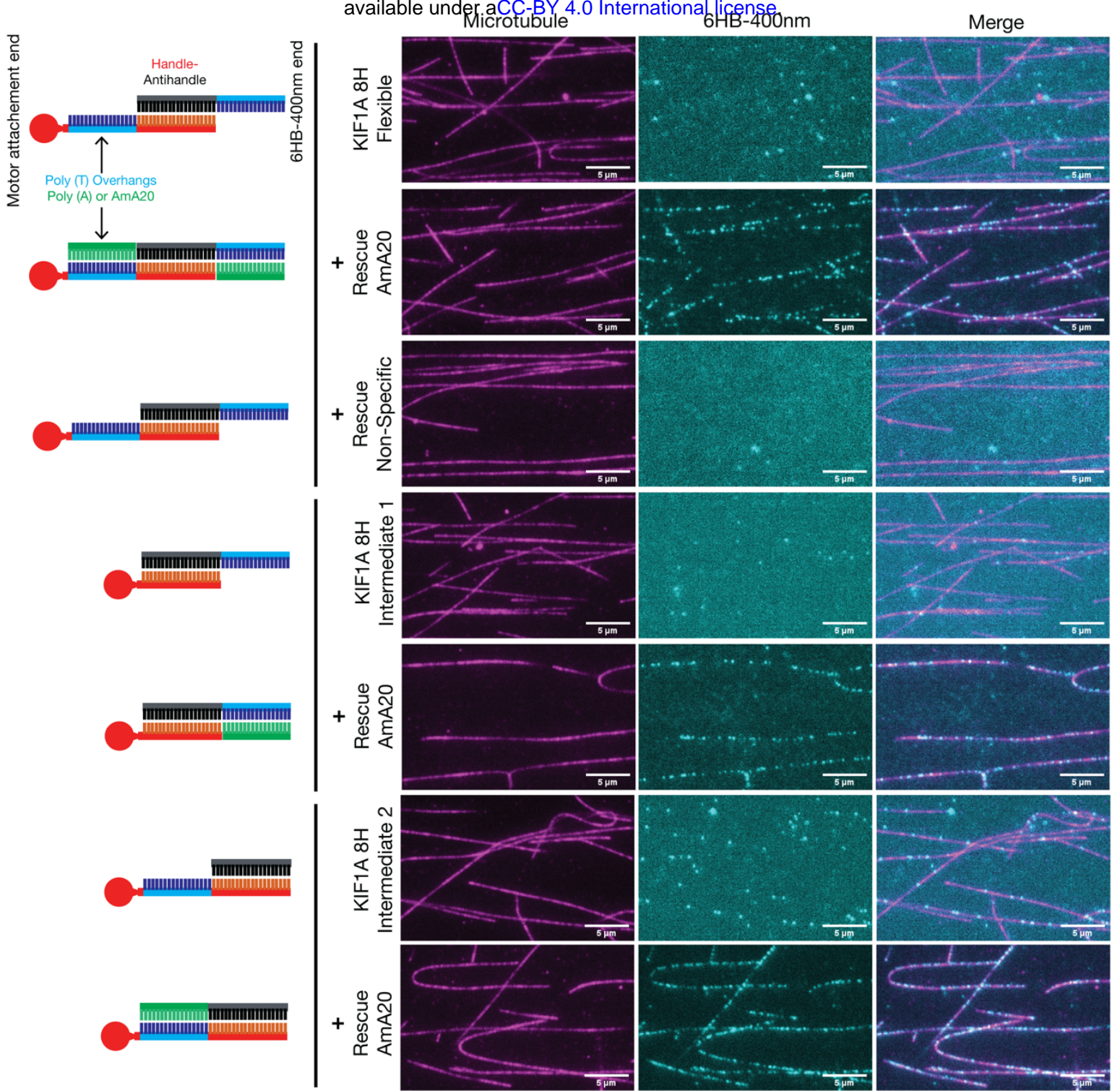

Figure 3: Microtubule-binding of KIF1A ensembles with varying linkers. Microtubule-binding of $6 \mathrm{HB}-400 \mathrm{~nm} 8 \mathrm{H}$ KIF1A monomer ensembles with varying linkers as indicated and illustrated. The microtubules are shown in magenta and the 6HB-400nm KIF1A $8 \mathrm{H}$ in cyan. In each microtubule-binding experiment, a rescue reaction was perfumed (marked as rescue), where an oligonucleotide that is complementary to the flexible parts of linkers was added to the mixture. For more details regarding the sequences of linkers and rescue oligonucleotides see Supplementary Table 1. Scale bar $=5 \mu \mathrm{m}$. 
bioRxiv preprint doi: https://doi.org/10.1101/2021.12.02.470904; this version posted December 2, 2021. The copyright holder for this preprint (which was not certified by peer review) is the author/funder, who has granted bioRxiv a license to display the preprint in perpetuity. It is made

A

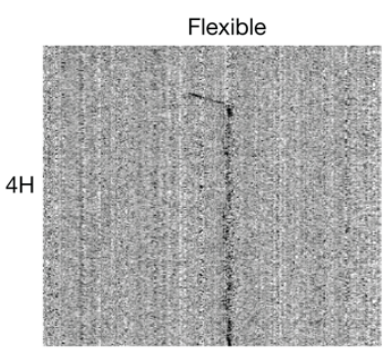
available under aCC-BY 4.0 International license.

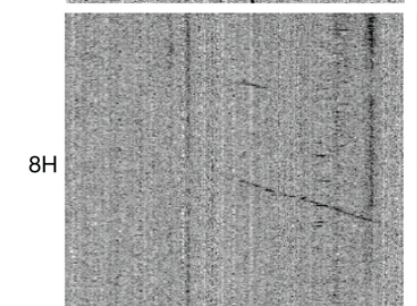

Rescue Oligonucleotides
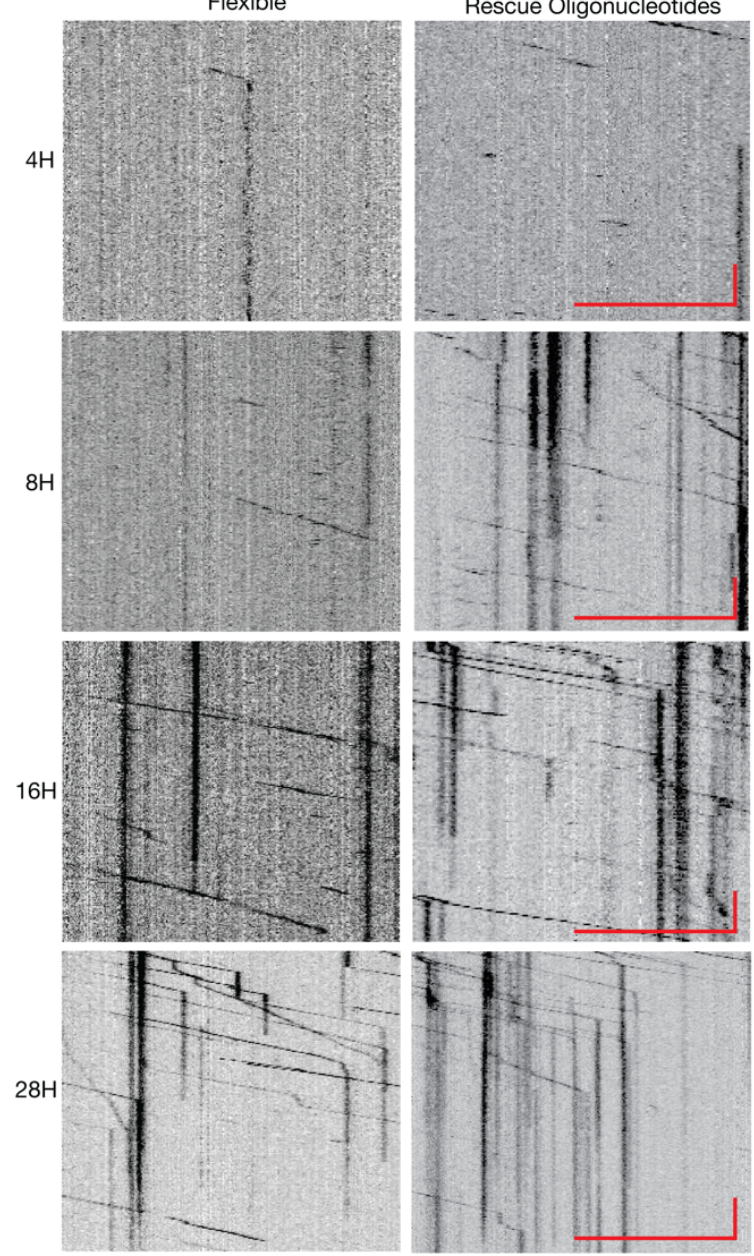

B

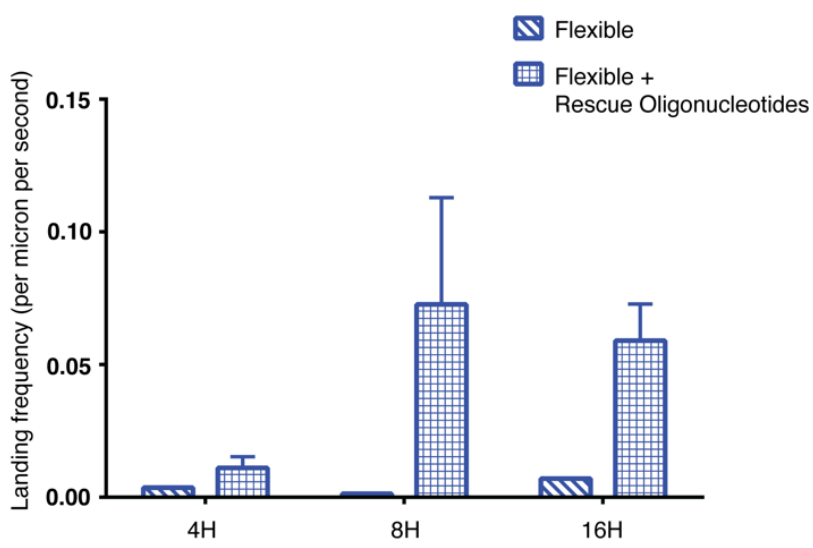

Figure 4: Motility properties of KIF1A ensembles with varying linkers. A. Representative kymographs of 6HB-400nm 4-28H KIF1A monomer ensembles with flexible and flexible-rescue oligonucleotides. Scale bar $=5 \mu \mathrm{m}$ and 10 seconds. B. Average landing frequency rates for $6 \mathrm{HB}-400 \mathrm{~nm} 4 \mathrm{H}, 8 \mathrm{H}$ and $16 \mathrm{H}$ KIF1A monomer ensembles with flexible linkers and rescue oligonucleotides as indicated. Error bars represent the standard error of the mean from three independent experiments. 
bioRxiv preprint doi: https://doi.org/10.1101/2021.12.02.470904; this version posted December 2, 2021. The copyright holder for this preprint (which was not certified by peer review) is the author/funder, who has granted bioRxiv a license to display the preprint in perpetuity. It is made A available under aCC-BY 4.0 International license.
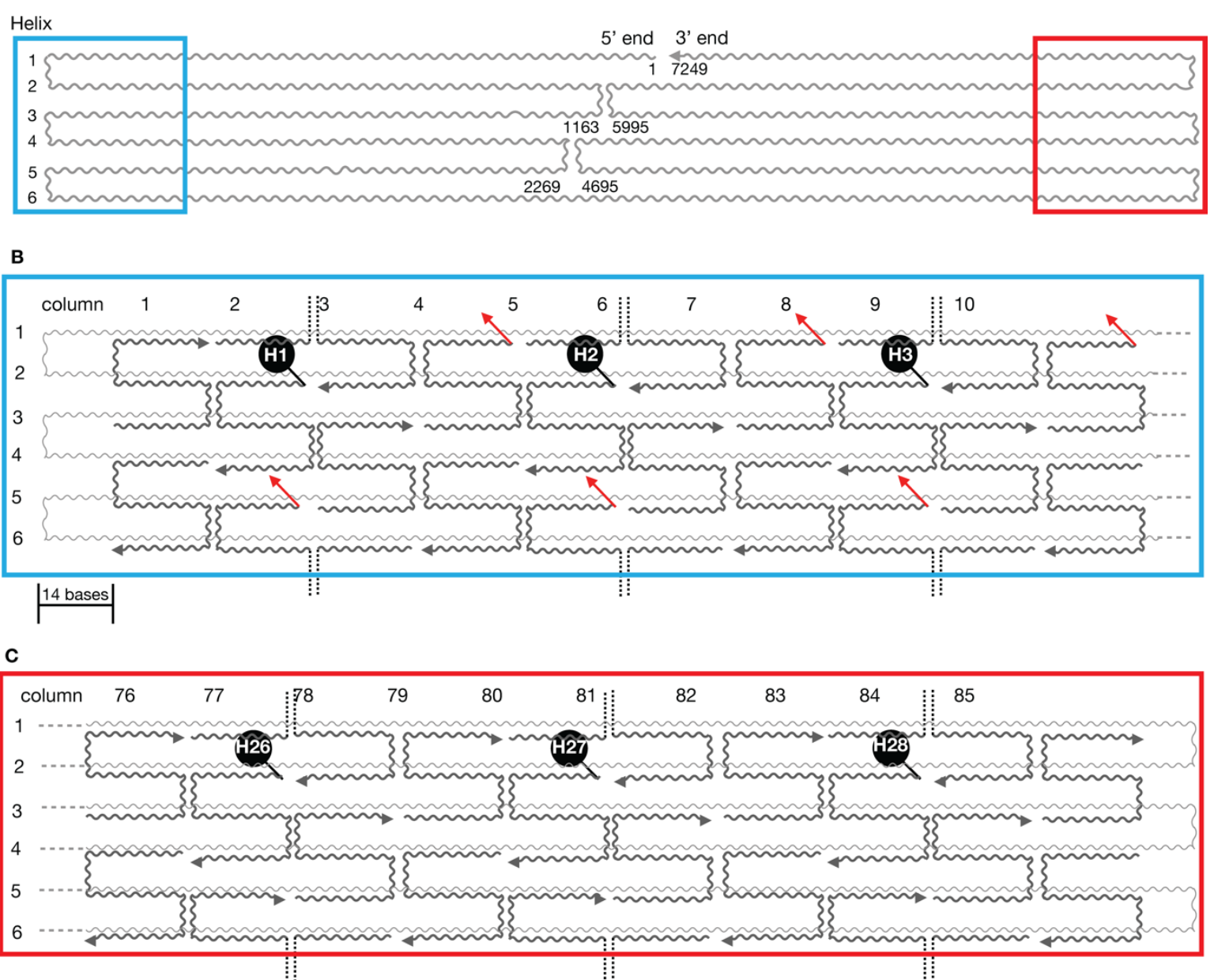

Supplementary Figure 1: Illustration of DNA scaffold with protein attachment and fluorescent handle sites. A. Arrangement of $13 \mathrm{mp} 18$ single-stranded DNA as 6HB-400nm DNA origami scaffold. The helices and corresponding base number are as indicated. B \& C. Zoomed in view of two distal end of $6 \mathrm{HB}-400 \mathrm{~nm}$ DNA origami scaffold as color coded in A. The m13mp18 strand and the complementary staple oligos are marked as wavy lines in light and dark grey respectively. The fluorescent handle extensions are indicated as red arrows. The helices, column number and handle numbers $(\mathrm{H} 1, \mathrm{H} 2, \mathrm{H} 3 \ldots \mathrm{H} 26, \mathrm{H} 27$ \& $\mathrm{H} 28)$ and nucleotide base resolution are as indicated. 
bioRxiv preprint doi: https://doi.org/10.1101/2021.12.02.470904; this version posted December 2, 2021. The copyright holder for this preprint (which was not certified by peer review) is the author/funder, who has granted bioRxiv a license to display the preprint in perpetuity. It is made

A

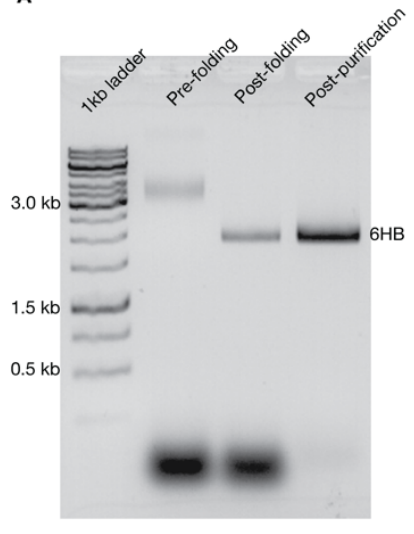

C

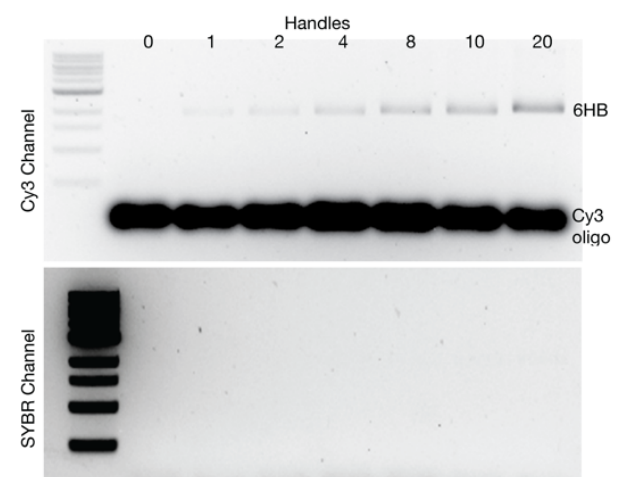

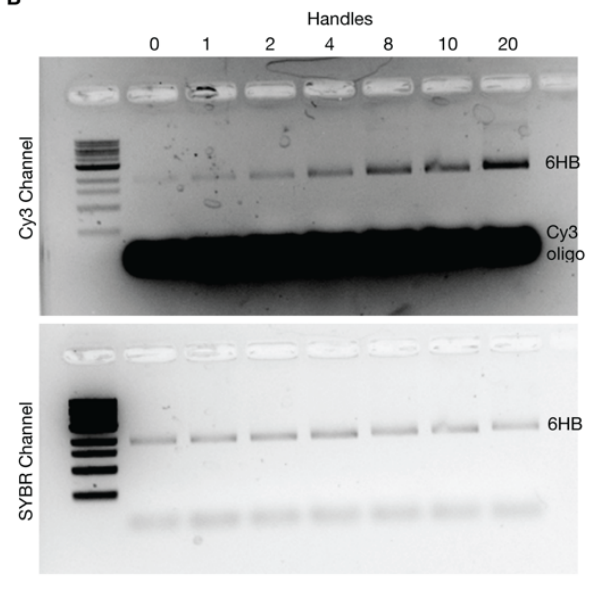

D

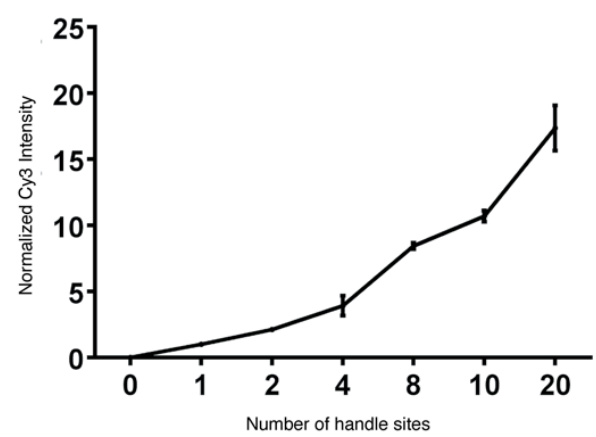

Supplementary Figure 2: Folding and validation of 400nm-6HB handle occupancy. A. Agarose gel of m13mp18 scaffold (pre-folding lane), folded reaction of $400 \mathrm{~nm}-6 \mathrm{HB}$ (post-folding lane) and $6 \mathrm{HB}-400 \mathrm{~nm}$ after removing the excess oligonucleotides (post-purification lane). The is marked as $6 \mathrm{hb}$ and the size of ladder as indicated. B. Agarose gel of $6 \mathrm{HB}-400 \mathrm{~nm}$ with varying handle numbers incubated Cy3-labelled anti-handle oligonucleotides as indicated, and the same gel was imaged under Cy3 and SYBR (UV) channel. C. Agarose gel of 6HB-400nm with varying handle numbers incubated $\mathrm{Cy} 3$-labelled anti-handle oligonucleotides as indicated. To rule out effects of the SYBR green, the experiments were performed without SYBR. The same gel was imaged under Cy3 and SYBR (UV) channel. D. Mean fold increase in $\mathrm{Cy} 3$ fluorescence as a function of handle number present in the 6HB-400nm DNA origami scaffold. Error bars represent the standard error of the mean from three independent experiments. 
bioRxiv preprint doi: https://doi.org/10.1101/2021.12.02.470904; this version posted December 2, 2021. The copyright holder for this preprint (which was not certified by peer review) is the author/funder, who has granted bioRxiv a license to display the preprint in perpetuity. It is made

A

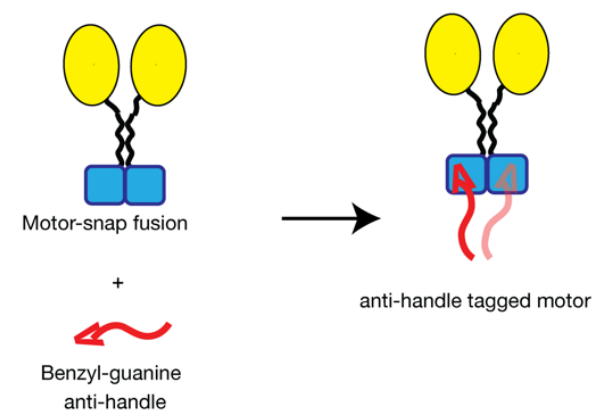

B

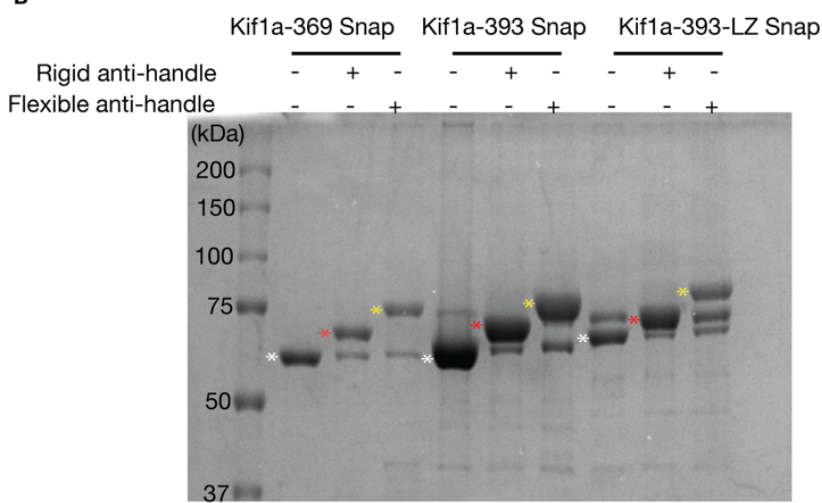

c
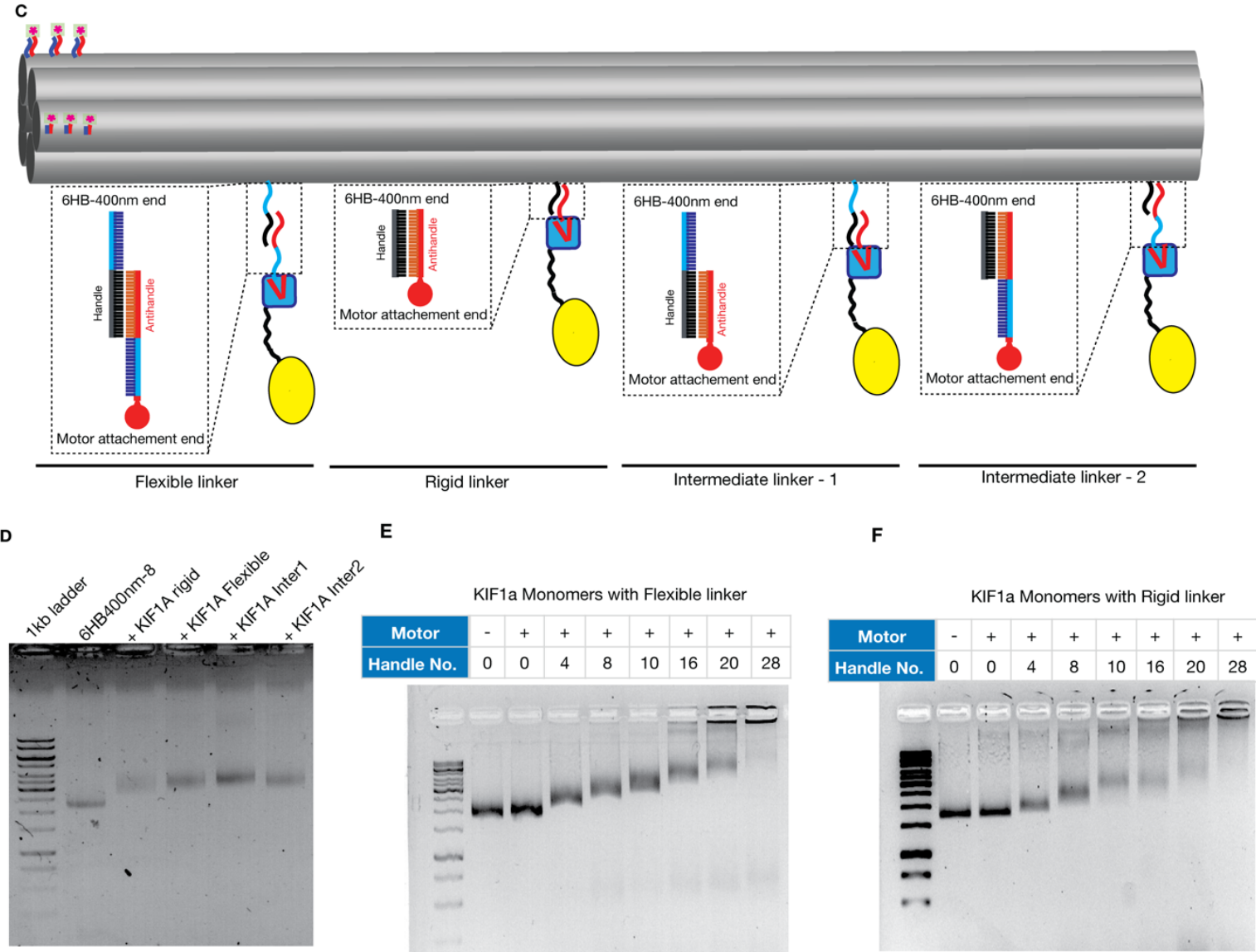

Supplementary Figure 3: Engineering kinesins for DNA attachment. A. Cartoon representation of KIF1A motors tagged with oligonucleotides using benzyl-guanine and SNAP tag method. B. SDS-PAGE gel of KIF1A monomer and dimer before and after tagged with flexible and rigid anti-handle oligonucleotides as indicated. The white, red and yellow asterisks denote KIF1A unconjugated, KIF1A conjugated with rigid and flexible oligonucleotides respectively. C. Illustration of 6HB-400nm DNA origami scaffold with flexible, rigid and intermediate motor linkers as indicated. For details regarding the sequences of oligonucleotides see Supplementary Table 1. D. Agarose gel shift assays for 6HB-400nm scaffold with and without KIF1A monomer ensembles for the $8 \mathrm{H}$ used in Figure 3 rigor experiments. E. \& F. Agarose gel shift assays for $6 \mathrm{HB}-400 \mathrm{~nm}$ scaffold with and without KIF1A monomers with varying handle sites for flexible and rigid linkers as marked. 


\section{Lama and Sirajuddin 2021}

bioRxiv preprint doi: https://doi.org/10.1101/2021.12.02.470904; this version posted December 2, 2021. The copyright holder for this preprint (which was not certified by peer review) is the author/funder, who has granted bioRxiv a license to display the preprint in perpetuity. It is made

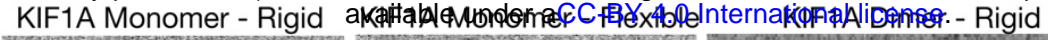

$4 \mathrm{H}$

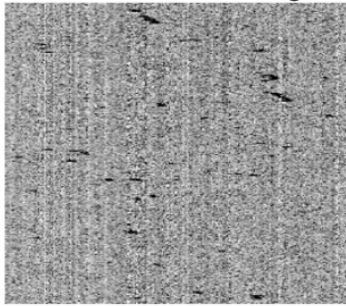

$8 \mathrm{H}$

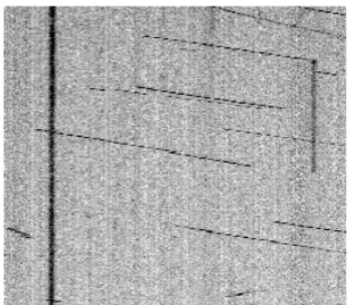

$6 \mathrm{H}$

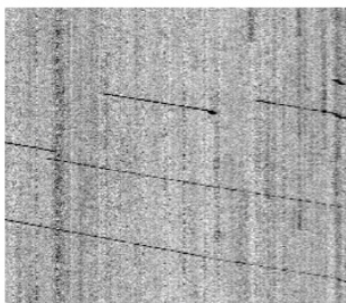

$28 \mathrm{H}$
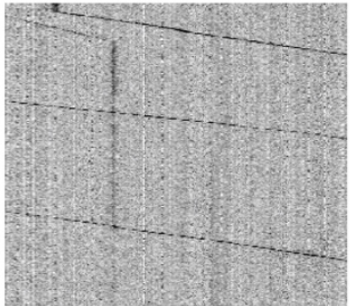
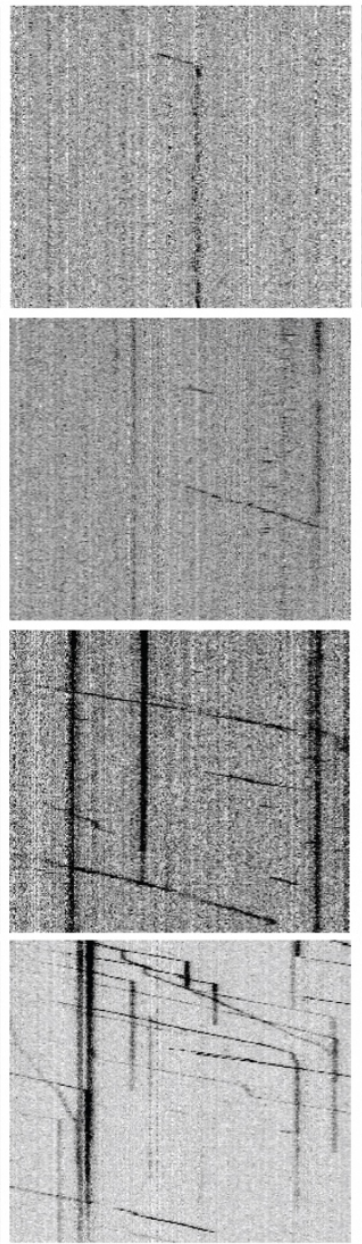
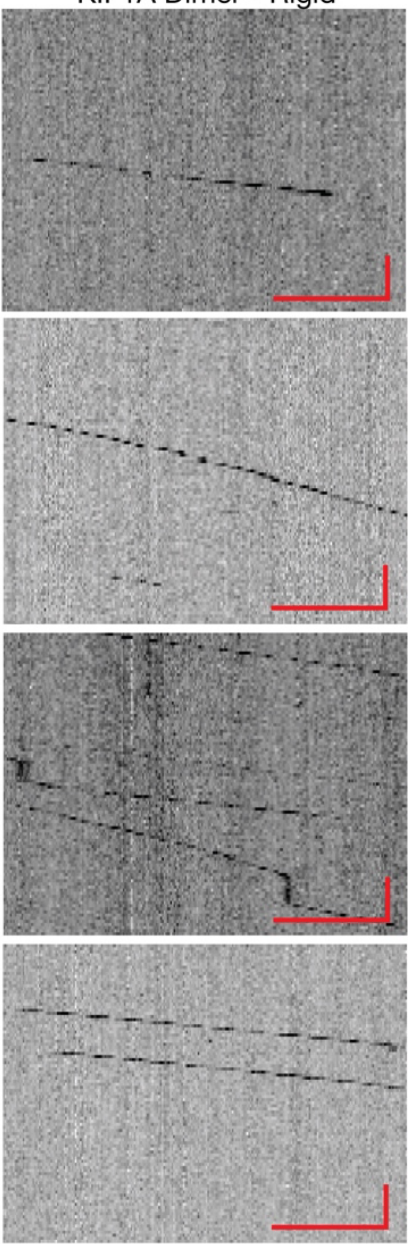

Supplementary Figure 4: Kymographs of KIF1A ensemble motility. Representative kymographs of KIF1A ensembles with flexible and rigid anti-handle oligonucleotides, for the data shown in Figure 2 as indicated. Scale bar $=5 \mu \mathrm{m}$ and 10 seconds. 
bioRxiv preprint doi: https://doi.org/10.1101/2021.12.02.470904; this version posted December 2, 2021. The copyright holder for this preprint (which was not certified by peer review) is the author/funder, who has granted bioRxiv a license to display the preprint in perpetuity. It is made

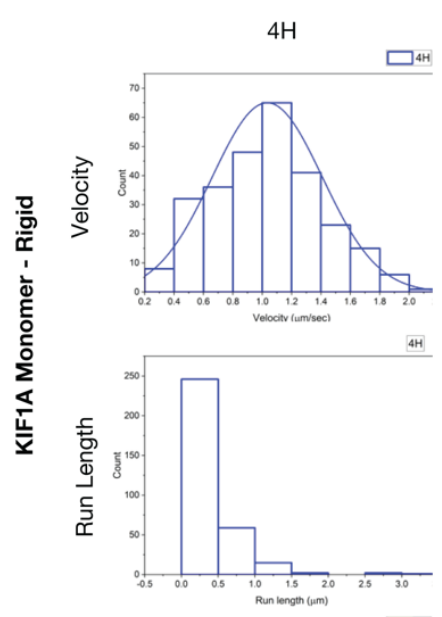
available under aCC-BY 4.0 International license.
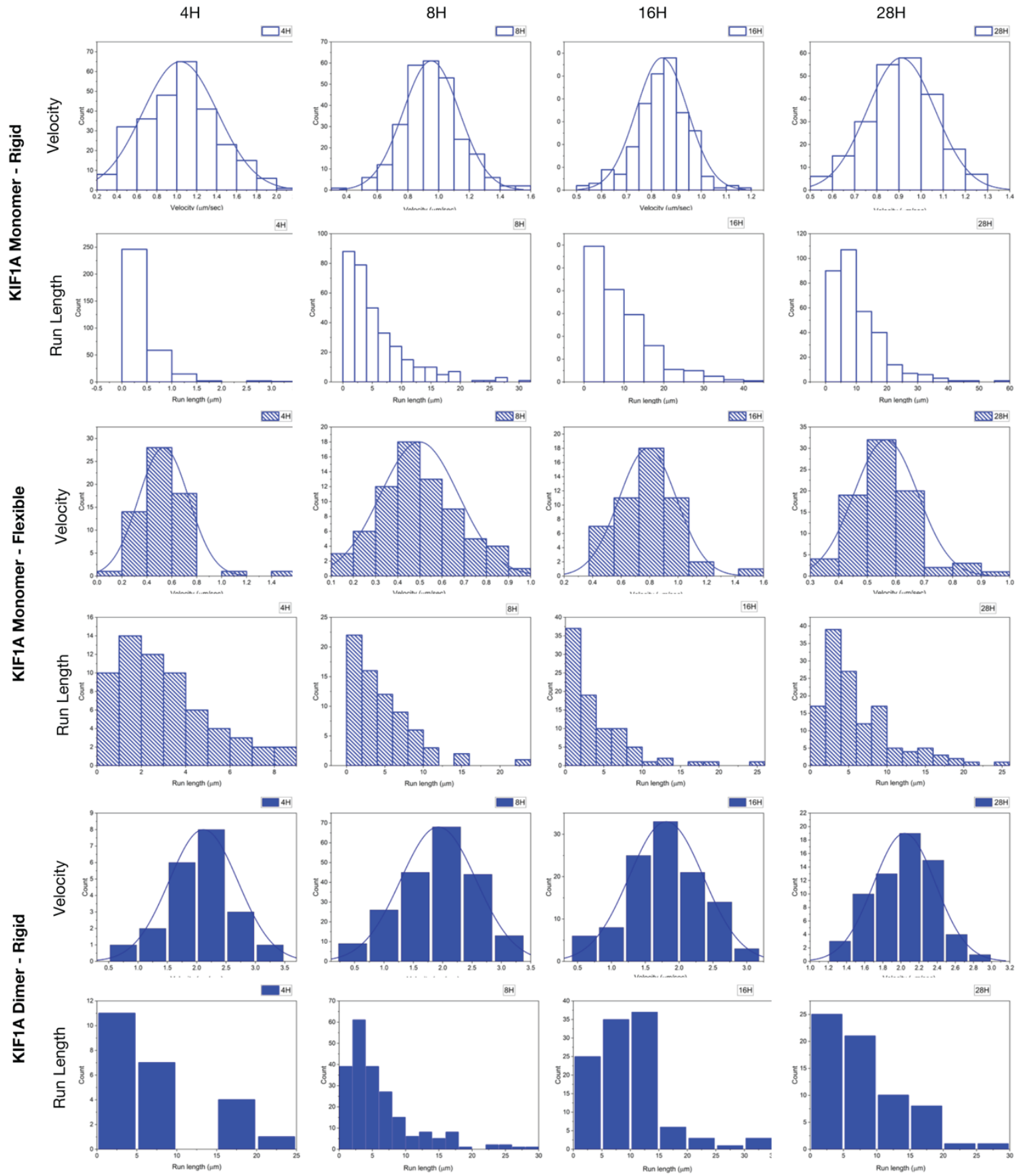

Supplementary Figure 5: Velocity and run length histograms of KIF1A ensemble motility. Velocity and processivity histograms of $6 \mathrm{HB}-400 \mathrm{~nm} 4-28 \mathrm{H}$ KIF1A monomer and dimer ensembles. Data for KIF1A monomer ensembles with flexible, rigid and KIF1A dimer ensembles with rigid anti-handle oligonucleotides are shown as indicated. 


\section{Lama and Sirajuddin 2021}

bioRxiv preprint doi: https://doi.org/10.1101/2021.12.02.470904; this version posted December 2, 2021. The copyright holder for this preprint (which was not certified by peer review) is the author/funder, who has granted bioRxiv a license to display the preprint in perpetuity. It is made

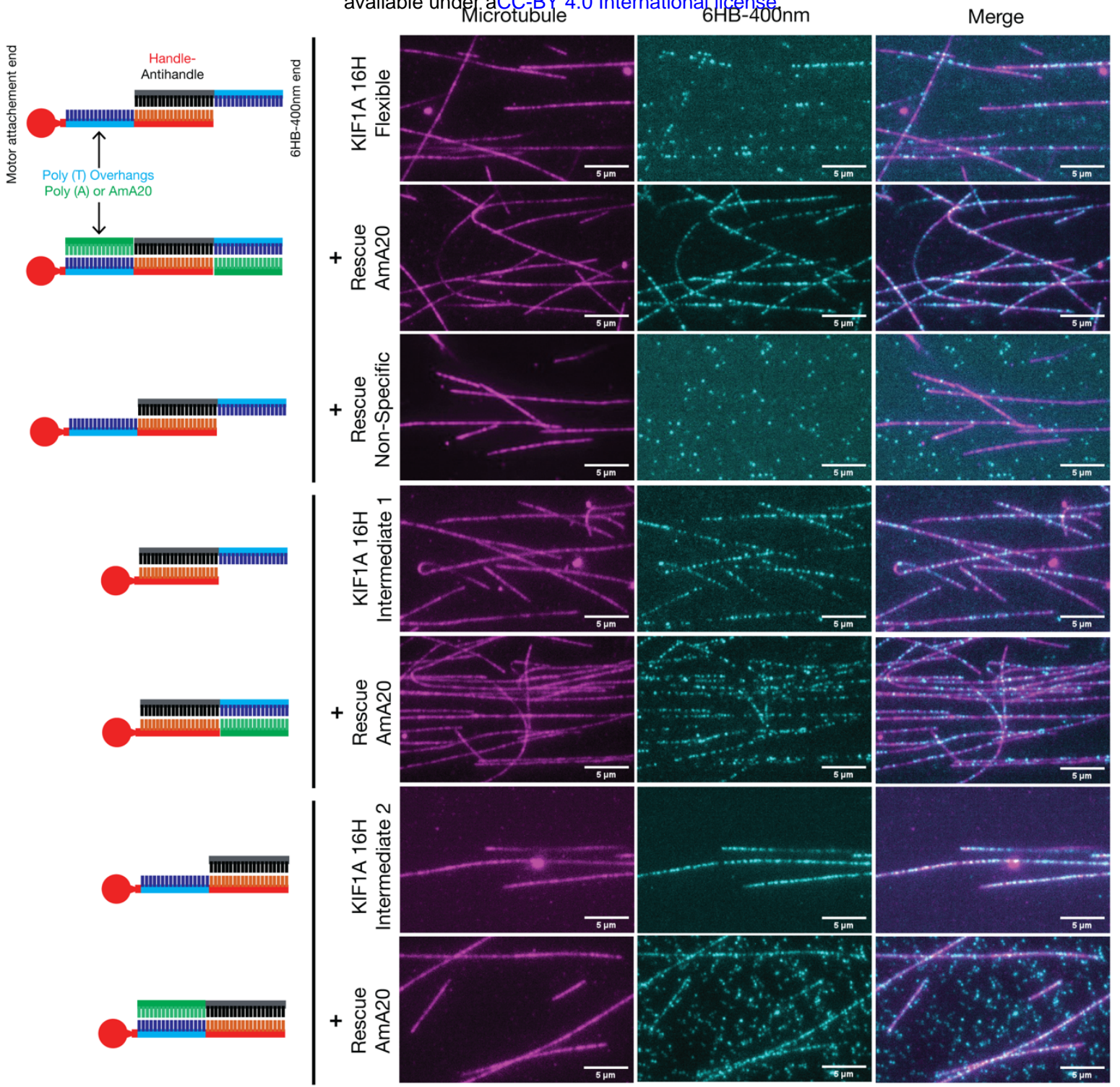

Supplementary Figure 6: Microtubule binding of KIF1A 16H ensembles. Microtubule-binding of $6 \mathrm{HB}-400 \mathrm{~nm} 16 \mathrm{H}$ KIF1A monomer ensembles with varying linkers as indicated and illustrated. The microtubules are shown in magenta and the 6HB-400nm KIF1A ensembles in cyan. In each microtubule-binding experiment, a rescue reaction was perfumed (marked as rescue), where an oligonucleotide that is complimentary to the flexible parts of linkers was added to the mixture. For more details regarding the sequences of linkers and rescue oligonucleotides see Supplementary Table 1. Scale bar $=5 \mu \mathrm{m}$. 
bioRxiv preprint doi: https://doi.org/10.1101/2021.12.02.470904; this version posted December 2, 2021. The copyright holder for this preprint (which was not certified by peer review) is the author/funder, who has granted bioRxiv a license to display the preprint in perpetuity. It is made $\underset{4 \mathrm{H} \text { rescue }}{\text { available under aCC-BY } 4.0 \text { International license. }}$ 4H rescue
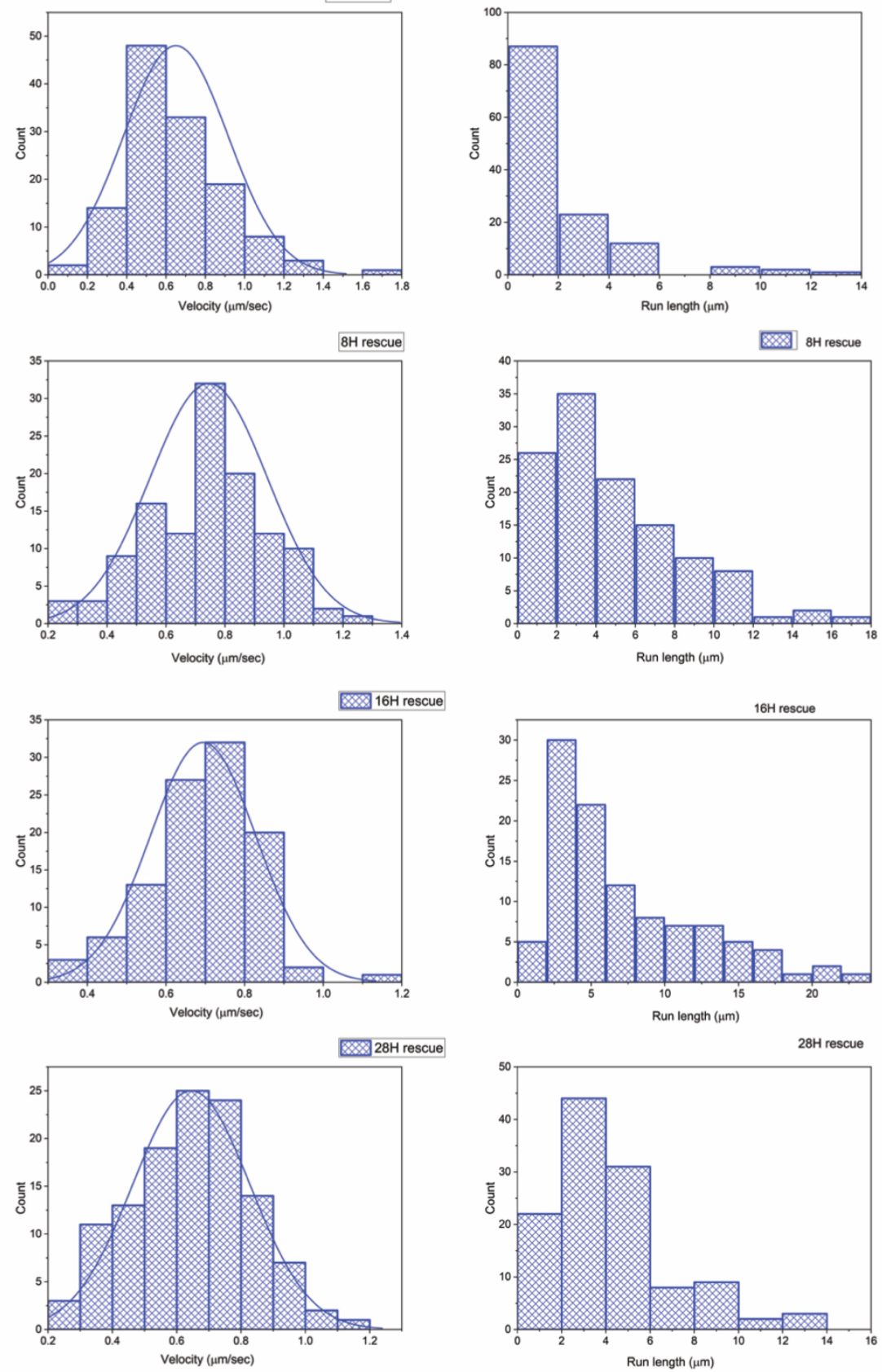

Supplementary Figure 7: Histograms and kymographs of KIF1A ensembles with flexible and rescue linkers. A. Velocity and processivity histograms of 6HB400nm 4-28H KIF1A monomer ensembles with flexible and flexible-rescue oligonucleotides, for the data shown in Figure 4. 